\title{
The Dirichlet Problem for a Class of Elliptic Difference Equations*
}

\author{
By G. T. McAllister
}

\begin{abstract}
Under suitable assumptions on the order of nonlinearity we prove existence and uniqueness theorems for difference Dirichlet problems of divergence type. We also show that the discrete solutions converge to a solution of the continuous problem. We do not assume that our equation comes from a variational problem. Some of our results are constructive or allow for the application of constructive methods.
\end{abstract}

Introduction. Let $\Omega$ be a plane bounded region such that the boundary of $\Omega$, $\partial \Omega$, is of class $C^{\mathbf{1}}$.

If $P$ is a point in the plane, then we denote it by $\left(x_{1}, x_{2}\right)$. Place a square grid on the plane of grid width $h$. All points of the form $(m h, n h)$, with $m$ and $n$ integers, are called mesh points. Let $P_{0}=\left(x_{01}, x_{02}\right)$ be a mesh point. Then a neighborhood of $P_{0}$ is the set of points $\Re\left(P_{0}\right)=\left\{\left(x_{01}, x_{02}\right),\left(x_{01}+h, x_{02}\right),\left(x_{01}+h, x_{02}+h\right),\left(x_{01}, x_{02}+h\right)\right.$, $\left.\left(x_{01}-h, x_{02}+h\right),\left(x_{01}-h, x_{02}\right),\left(x_{01}-h, x_{02}-h\right),\left(x_{01}, x_{02}-h\right),\left(x_{01}+h, x_{02}-h\right)\right\}$. We define $\Omega_{h}$ as that set of mesh points $P$ such that $\mathscr{N}(P) \subset \bar{\Omega}$, and we define the boundary of $\Omega_{h}$ as those mesh points $P$ in $\bar{\Omega}$ such that at least one element of $\mathfrak{T}(P)$ is in the exterior of $\bar{\Omega}=\Omega+\partial \Omega$.

Let $V(P)$ be any function which is everywhere finite for $P \in \Omega_{h}+\partial \Omega_{h}=\bar{\Omega}_{h}$; such a function will be called a mesh function. Let $P=\left(x_{1}, x_{2}\right)$ be a mesh point. Then, for any mesh function we define forward difference quotients by

$$
V_{x_{1}}(P)=\left\{V\left(x_{1}+h, x_{2}\right)-V(P)\right\} / h, \quad V_{x_{9}}(P)=\left\{V\left(x_{1}, x_{2}+h\right)-V(P)\right\} / h
$$

and backward difference quotients by

$$
V_{t_{1}}(P)=\left\{V(P)-V\left(x_{1}-h, x_{2}\right)\right\} / h, \quad V_{x_{2}}(P)=\left\{V(P)-V\left(x_{1}, x_{2}-h\right)\right\} / h .
$$

The vector $\left(V_{x_{1}}(P), V_{x_{2}}(P)\right)$ is denoted by $\nabla_{h} V(P)$ and the vector $\left(V_{t_{1}}(P), V_{x_{2}}(P)\right)$ is denoted by $\bar{\nabla}_{h} V(P)$.

We denote by $\Omega_{h}^{\prime}$ (and $\Omega_{h}^{\prime \prime}$ ) the set of points $P \in \bar{\Omega}_{h}$ such that, for any mesh function $V(P)$ defined on $\bar{\Omega}_{h}$, the vector $\nabla_{h} V(P)$ (and $\bar{\nabla}_{h} V(P)$ ) is defined using only mesh points in $\bar{\Omega}_{h}$. If $D_{h}$ is any set of mesh points in the plane, then we define $m_{h}\left(D_{h}\right)$ to be $h^{2}$ times the number of points in $D_{h}$. A set of mesh points will be called connected iff one can go from any mesh point in the set to any other mesh point in the set along line segments of length $h$ connecting only elements of the set. We assume all mesh sets are connected.

A function $u(P) \in C_{c}^{p}(\Omega)$ iff the support of $u(P)$ is a compact subset of $\Omega$ and all $p$ th order partial derivatives of $u(P)$ are continuous over $\Omega$. The set $\mathscr{L}_{m}(\Omega)$ denotes all

Received December 2, 1969, revised December 3, 1970.

AMS 1970 subject classifications. Primary 39A10; Secondary 35J60.

Key words and phrases. Elliptic difference equations, divergence type, approximation of solution, boundary-value problems, convergence of solution, existence, uniqueness criteria.

* Research supported by contract GP 7192 with the National Science Foundation. 
functions whose absolute value is Lebesgue integrable to the $m$ th power. The space $\mathcal{F}_{m, 0}^{p}(\Omega)$ is the completion of $C_{c}^{p}(\Omega)$ with respect to the metric of $\mathfrak{L}_{m}(\Omega)$ applied to all partial derivatives up to order $p$; for a more detailed discussion see Morrey [12, pp. 62-90].

In this paper, we shall study selfadjoint uniformly elliptic differential problems of the form

$$
\begin{aligned}
\left(a_{i}(P, u(P), \nabla u(P))\right)_{x_{i}} & =f(P, u(P), \nabla u(P)), \quad P \in \Omega, \\
u(P) & =q(P), \quad P \in \partial \Omega,
\end{aligned}
$$

where the repeated indices are summed and $\nabla u(P)=\left(u_{x_{1}}(P), u_{x},(P)\right)$ with $u_{x_{i}}(P)=$ $\partial u(P) / \partial x_{i}$. We consider the difference approximation associated with this equation for given mesh size $h$ to be given as

$$
\begin{aligned}
\left(a_{i}\left(P, U, \nabla_{h} U\right)\right)_{\dot{x}_{i}} & =f\left(P, U, \nabla_{h} U\right), \quad P \in \Omega_{h}, \\
U(P) & =Q(P), \quad P \in \partial \Omega_{h} .
\end{aligned}
$$

The mesh function $Q(P)$ is related to $q(P)$ in that we assume $q(P)$ has a nice extension to $\Omega$; for our purposes it is sufficient to assume that it may be extended to $\Omega$ as an element of $C^{2}(\bar{\Omega})$, call it $\tilde{q}(P)$, and we define $Q(P)=\tilde{q}(P)$ for $P \in \partial \Omega_{h}$.

The results of this paper will also hold for the difference problem

$$
\begin{aligned}
\left(a_{i}\left(P, U, \nabla_{h} U\right)\right)_{\bar{x}_{i}}+\left(a_{i}\left(P, U, \bar{\nabla}_{h} U\right)\right)_{x_{i}} & \\
& =f\left(P, U, \nabla_{h} U\right)+f\left(P, U, \bar{\nabla}_{h} U\right), \quad P \in \Omega_{h}, \\
U(P) & =Q(P), \quad P \in \partial \Omega_{h} .
\end{aligned}
$$

Let $Q_{0}\left(\Omega_{h}\right)$ be the set of all mesh functions defined on $\Omega_{h}$ and such that they vanish on $\partial \Omega_{h}$. Any solution $U(P)$ of $\left({ }^{* *}\right)$ will be such that, for every $\zeta \in a_{0}\left(\Omega_{h}\right)$,

$$
h^{2} \sum_{\Omega_{h^{\prime}}}\left\{a_{i}\left(P, U, \nabla_{h} U\right) \zeta_{x_{i}}+f\left(P, U, \nabla_{h} U\right) \zeta\right\}=0 .
$$

To save space, we shall often drop the index set of the summation. If we used the approximation in $\left({ }^{* *}\right)^{\prime}$, we would add to $(* * *)$ a summation over $\Omega_{h}^{\prime \prime}$. It is this last equation we shall study when we prove results of existence, uniqueness and convergence.

It is clear that to prove conditions for existence, uniqueness and convergence, we must make some assumptions which describe in a gross sense the types of nonlinearity we are considering in $\left(^{*}\right)$. We list these classical assumptions as follows (for a more detailed analysis of the genesis of these conditions see [7] and [12]):

Condition (A). There exists a nonnegative constant $C_{1}$ such that, for mesh functions $V(P)$ and $W(P)$,

$$
\sum_{i=1}^{2} a_{i}\left(P, V(P), \nabla_{h} W(P)\right) p_{i}(P) \geqq C_{1}\left|\nabla_{h} W(P)\right|^{m},
$$

where $p_{i}(P)=W_{x_{i}}(P)$ for $i=1,2$ and $P \in \Omega_{h}$.

Condition (B). There exist nonnegative constants $C_{2}$ and $C_{3}$ such that for any nonzero vector $\xi=\left(\xi_{1}, \xi_{2}\right)$, for any $P \in \Omega_{h}$ and for any mesh functions $V(P)$ and $W(P)$, we have 


$$
\begin{aligned}
C_{3}\left\{\left|\nabla_{h} W(P)\right|^{2}+1\right\}^{(m-2) / 2}|\xi|^{2} & \geqq \frac{\partial a_{i}\left(P, V(P), \nabla_{h} W(P)\right)}{\partial p_{i}} \xi_{i} \xi_{i} \\
& \geqq C_{2}\left\{\left|\nabla_{h} W(P)\right|^{2}+1\right\}^{(m-2) / 2}|\xi|^{2},
\end{aligned}
$$

where $a_{i, p_{i}}=a_{i, p_{i}},|\xi|^{2}=\xi_{1}^{2}+\xi_{2}^{2}$ and the repeated indices indicate a summation over all $i$ and $j$. This condition includes the statement that the problem in (1) is elliptic.

Condition (C). There exists a nonnegative constant $C_{4}$ such that

$(\alpha)\left|f\left(P, V(P), \nabla_{h} W(P)\right)\right| \leqq C_{4}\left\{1+\left|\nabla_{h} W\right|^{2}\right\}^{(m-1) / 2}$, or

(B) $\left|f\left(P, V(P), \nabla_{h} W(P)\right)\right| \leqq C_{4}\left\{1+\left|\nabla_{h} W\right|^{2}\right\}^{(m-1-\kappa) / 2}$, with $\kappa>0$.

Condition (D). There exist nonnegative constants $C_{5}, C_{6}$ such that for mesh functions $V(P), W(P)$ we have the relations

$$
\sum_{i}\left\{\left|\partial a_{i}\left(P, V, \nabla_{h} W\right) / \partial x_{i}\right|+\left|a_{i}\left(P, V, \nabla_{h} W\right)\right|\right\} \leqq C_{5}\left(1+\left|\nabla_{h} W\right|^{2}\right)^{(m-1) / 2}
$$

and

$$
\sum_{i}\left|\partial a_{i}\left(P, V, \nabla_{h} W\right) / \partial V\right| \leqq C_{6}\left(1+\left|\nabla_{h} W\right|^{2}\right)^{(m-2) / 2} .
$$

Condition (E). There exists a nonnegative constant $C_{7}$ such that one of the following is valid:

$$
\left|\partial f\left(P, V(P), \nabla_{h} W(P)\right) / \partial V\right|
$$

$(\alpha)$

$$
\begin{aligned}
& +\sum_{i}\left\{\left|\partial f\left(P, V(P), \nabla_{h} W(P)\right) / \partial W_{x_{i}}\right|+\left|\partial f\left(P, V(P), \nabla_{h} W(P)\right) / \partial x_{i}\right|\right\} \\
\leqq & C_{7}\left(1+\left|\nabla_{h} W\right|^{2}\right)^{(m-2) / 2},
\end{aligned}
$$$$
\left|\partial f\left(P, V(P), \nabla_{h} W(P)\right) / \partial V\right|
$$

$$
\begin{aligned}
& +\sum_{i}\left\{\left|\partial f\left(P, V(P), \nabla_{h} W(P)\right) / \partial W_{x_{i}}\right|+\left|\partial f\left(P, V(P), \nabla_{h} W(P)\right) / \partial x_{i}\right|\right\} \\
\leqq & C_{7}\left(1+\left|\nabla_{h} W\right|^{2}\right)^{(m-2-\kappa) / 2}
\end{aligned}
$$

where $\kappa>0$, or

$$
\left|\partial f\left(P, V(P), \nabla_{h} W(P)\right) / \partial V\right|+\sum_{i}\left|\partial f\left(P, V(P), \nabla_{h} W(P)\right) / \partial W_{x_{i}}\right|=0
$$

and

$$
h^{2} \sum_{\bar{\Omega}_{h}}|f(P)| \leqq C_{7}, \text { for } m>2,
$$

or

$$
h^{2} \sum_{\bar{\Omega}_{h}}|f(P)|^{m} \leqq C_{7}, \quad \text { for } m \leqq 2 .
$$

We are assuming that $m>1$ in all of these conditions and that all the constants $C_{1}, C_{2}$, etc. are independent of $h$. By being independent of $h$ we mean that if we imagine that $h$ goes to zero then these numbers are to remain finite in the limit.

We shall use the notation, in order to save space in the sequel, $m^{\prime}$ and $\bar{m}$ where

$$
m^{\prime}=m-1 \text { and } \bar{m}=m-2 .
$$


We shall also consider problems where we replace the expression $1+\left|\nabla_{h} W\right|^{2}$ by the expression $1+|V|^{2}+\left|\nabla_{h} W\right|^{2}$ in the appropriate conditions above. When we do this we shall denote the conditions corresponding to (A), (B), etc. by (A'), (B'), etc.

Ladyženskaya and Ural'ceva [8, p. 230] have considered the case that the inhomogeneous term in the differential equation associated with (1) satisfies the condition that $|f(P, u, \nabla u)| \leqq C_{4}\left(1+|\nabla u|^{2}\right)^{m / 2}$. Their development is relative to the max norm, ours is not, and their analysis rests heavily on the Dirichlet Growth Theorem of Morrey [12, p. 79] and on the extension of results of De Giorgi [12, p. 194]. We can prove some of these preliminary results but when we attempt to apply them to a solution of $\left({ }^{* *}\right)$, by a summation by parts, we cannot use these results because on the $\partial A_{h, k, \rho}, A_{h, k, \rho}=\left\{P: P \in \bar{\Omega}_{h}, U(P)>k,\left|P-P_{0}\right|<\rho\right\}$, we do not have that $U(P)=k$.

There is one more condition that we will add in order to prove the existence of a solution to $(* * *)$ over general domains.

Condition (F). For any mesh function $\xi(P) \in Q_{0}\left(\Omega_{h}\right)$ and for every mesh function $W(P) \in a_{0}\left(\Omega_{h}\right)$, there exists a function $F\left(P, \xi(P), \nabla_{h} W(P)\right)$ such that

$$
a_{i}\left(P, \xi(P), \nabla_{h} W(P)\right)=\partial F\left(P, \xi(P), \nabla_{h} W(P)\right) / \partial W_{x_{i}}(P)
$$

and

$$
a_{i}\left(P, \xi(P), \bar{\nabla}_{h} W(P)\right)=\partial F\left(P, \xi(P), \bar{\nabla}_{h} W(P)\right) / \partial W_{x_{i}}(P) .
$$

We remark that this Condition (F) does not say that our equation in $(* *)$ is the Euler equation of a variational problem. Hence, our condition in $(F)$ is more general than a requirement of Frehse [3, p. 316] who assumes that his equations come from a variational problem. We will also consider a sufficient condition for removing Condition (F).

We also consider the special case that $\Omega$ is a rectangular region with sides parallel to the coordinate axes. In this case, we assume that the lengths of two adjacent sides are commensurable and that $\partial \Omega_{h} \subset \partial \Omega$. We denote the sides of $\Omega$ by $s_{i}, i=1, \cdots, 4$, with $s_{1}$ on the side perpendicular to the $x_{1}$-axis and the ordering increasing in the counterclockwise direction. The rectangular region offers simplicities which are not present in any other region.

In this paper, we show, under certain constraints on the constants $C_{i}$ of our conditions, that a solution to $(* *)$ exists; the problem in $(* *)$ is equivalent to that in $(* * *)$. The proof of the existence of a solution is given in Theorem 1 and it makes essential use of the Brouwer Fixed Point Theorem. To apply that theorem, we must prove, in part, that a certain function, called $\phi$, defined on a ball $S_{1}$ in $H_{m, 0}^{1}\left(\Omega_{h}\right)$, maps $S_{1}$ into itself. By requiring $C_{1}^{m}>C_{4}^{m} C_{8} 2^{m^{\prime} m^{\prime}}$ or $C_{1}^{m}>C_{4}^{m} 3^{m^{\prime} m^{\prime}} C_{8}\left(C_{8}^{m^{\prime} / m}+1\right)^{m}$, along with other conditions in the various parts of Theorem 1 , we are able to show that a $J_{1}>0$ exists so that for $S_{1}$ of radius $\geqq J_{1}$ the mapping property is satisfied. We also prove that this $\phi$ is Hölder-continuous on $\delta_{1}$ for $m>2$ and $\phi$ is Lipschitz-continuous on $S_{1}$ for $m \in[4 / 3,2)$. These continuity properties allow, under certain assumptions, the application of constructive methods; e.g. the Banach Fixed Point Theorem or some of the approximation methods in [16]. We also obtain certain uniqueness results from these methods. The special case $m=2$ is presented in Theorem 2 .

For the proof of Theorem 1 we assumed that Condition (F) holds. In Theorem 3, we present a sufficient condition-again based on the size of the constants $C_{i}$-for removing that condition. 
In Theorem 4, we obtain "interior estimates" on the norm of second-order difference quotients for solutions to $(* * *)$; these estimates hold up to the boundary if $\Omega$ is a rectangular region and if the expressions $a_{i}$ satisfy a condition given in Corollary 2.

In our proof of convergence, Theorem 5, we make essential use of Theorem 4 and the fact that we have two independent variables. In this respect, our proof of convergence is different from that of [3] and [5], although a more general proof of convergence is given in [3]. We show that for certain mesh sizes $h_{n}$ tending to zero, those associated solutions $U_{n}$ of $(* * *)$ may be extended to $\mathcal{U}_{n} \in \mathcal{F C}_{m, 0}^{1}(\Omega)$ such that a subsequence converges weakly to an element $\mathcal{u}_{0} \in \mathcal{F}_{m, 0}^{1}(\Omega)$ and strongly to $\mathcal{u}_{0}$ over $D^{\prime}$ where $\bar{D}^{\prime} \subset \Omega$ and the $\partial \Omega, \partial D^{\prime}$ are in $C^{1}$. From this and the fact that $\mathcal{u}_{n}=U_{n}$ over $D_{h_{n}}^{\prime}$, we conclude that $\mathcal{U}_{0}$ is a weak solution to $\left(^{*}\right)$. This result is strengthened in the case $\Omega$ is a rectangle; that result is given in Corollary 3 .

All of the results mentioned above were explicitly derived under the assumption that $Q(P) \equiv 0$ for $P \in \partial \Omega_{h}$. In part (I) of the paper, we mention how this assumption may be removed and the resulting effects on our computations become obvious.

Our assumption that the number $n$ of independent variables is two was only important in Theorem 5. All results, with the exception of this, go through for $n>2$ with slight modification. The proof of Theorem 5 would require a constraint on the relationship between $m$ and $n$.

The author is grateful to the referee for his careful reading of the original manuscript.

Existence, Uniqueness and Convergence. In this section, we shall consider uniformly elliptic difference problems of the form

$$
\begin{aligned}
\left(a_{1}\left(P, U(P), \nabla_{h} U(P)\right)\right)_{\dot{x}_{1}} & +\left(a_{2}\left(P, U(P), \nabla_{h} U(P)\right)\right)_{x_{2}} \\
& =f\left(P, U(P), \nabla_{h} U(P)\right), \quad P \in \Omega_{h}, \\
U(P) & =Q(P), \quad P \in \partial \Omega_{h} .
\end{aligned}
$$

A more general type of problem has been treated in [9] where $\Omega$ was a rectangular region; the geometry of $\Omega$ was essential to the methods developed in that paper. Here, we wish to specialize the equation to be of divergence form but to leave the geometry of $\Omega$ as broad as possible.

In the development of this section, we shall refer to Condition (A), Condition $\left(A^{\prime}\right)$, etc., and by this we shall mean those conditions given in the Introduction which describe the types of nonlinearity we are considering.

We now divide this section into two parts.

(I). The Case that $Q(P) \equiv 0$ for $P \in \partial \Omega_{h}$. Let $A_{0}\left(\Omega_{h}\right) \equiv a_{0}=\{\xi(P): \xi(P)$ is a finite mesh function defined on $\bar{\Omega}_{h}$ and $\xi(P)=0$ for $\left.P \in \partial \Omega_{h}\right\}$. Then, we define a weak solution to (1) by following Ladyženskaya [7, p. 91], as a solution $U(P)$ to the problem

$$
h^{2} \sum_{P \in \Omega_{h}}\left\{\sum_{i=1}^{2}\left(a_{i}\left(P, U(P), \nabla_{h} U(P)\right)\right)_{i_{i}}+f\left(P, U(P), \nabla_{h} U(P)\right)\right\} \zeta(P)=0,
$$

where $U(P) \in a_{0}$ and (2) is to hold for all $\zeta(P) \in a_{0}$. The Theorem of Gauss, see von Koppenfels [6, p. 10] or Cryer [2, p. 160], allows us to write (2) as: (repeated indices indicate summation over $i$ ) 


$$
h^{2} \sum_{P \in \mathbb{B}_{h}}\left\{a_{i}\left(P, U(P), \nabla_{h} U(P)\right) \zeta_{x_{i}}(P)-f\left(P, U(P), \nabla_{h} U(P)\right) \zeta(P)\right\}=0 ;
$$

here $\Omega_{h}^{\prime}$ is the subset of $\bar{\Omega}_{h}$ described in the Introduction.

Let $H_{m, 0}^{1}\left(\Omega_{h}^{\prime}\right)=\left\{V(P): V(P) \in a_{n}\right.$ and there exists a constant $K_{1}$ such that

$$
\|V(P)\|_{m, 0}^{1} \equiv\left(h^{2} \sum_{P \in \Omega_{h^{\prime}}}|V(P)|^{m}\right)^{1 / m}+\left(h^{2} \sum_{P \in \Omega_{h^{\prime}}}\left|\nabla_{h} V(P)\right|^{m}\right)^{1 / m} \leqq K_{1}
$$

and $K_{1}$ may be bounded, independent of mesh size $h$ \}. If we have two different mesh widths $h_{1}$ and $h_{2}$, then the domain of definition of the function $V(P) \in H_{m, 0}^{1}\left(\Omega_{h_{1}}^{\prime}\right)$ is different from the domain of definition of the function $V(P) \in H_{m, 0}^{1}\left(\Omega_{h_{2}}^{\prime}\right)$, because $\Omega_{h_{1}}^{\prime} \neq \Omega_{h_{2}}^{\prime}$. We shall not dwell on this idea until we consider the question of convergence. Suffice it to say at this point that we are taking $h$ to be arbitrarily small but fixed.

For any mesh function $W(P) \in Q_{0}$, we define the $l_{m}$-norm as $\|W(P)\|_{m}^{0}=$ $\left(h^{2} \sum_{P \in \Omega_{h}}|W(P)|^{m}\right)^{1 / m}$.

In the sequel, we shall drop the prime from $\Omega_{h}$ as it does not add to the exposition; our meanings will be clear from the context.

We shall now state some lemmas of the Sobolev type which are essential for the technical manipulations which will follow. Their proofs proceed exactly as in [10], [11], [12, p. 80], [7, p. 82] and [14, p. 10].

LEMMA 1. If $V(P) \in H_{m, 0}^{1}\left(\Omega_{h}\right), m \geqq 1$, and $\Omega_{h}$ is bounded and connected, then $h^{2} \sum_{\Omega_{h}}|V(P)|^{m} \leqq C_{8} h^{2} \sum_{\Omega_{h}}\left|\nabla_{h} V(P)\right|^{m}$ where $C_{8} \leqq 2 \max \left\{d_{x_{1}}^{m}, d_{x_{2}}^{m}\right\} \equiv 2 d^{m}$ with $d_{x_{i}}$ the maximum width of $\Omega_{h}$ in the $x_{i}$-direction.

LEMMA 2. If $\xi(P) \in H_{m, 0}^{1}\left(\Omega_{h}\right)$ with $1 \leqq m<2$, then $\xi(P) \in l_{r}\left(\Omega_{h}\right)$ where $r=2 m /(2-m)$ and $\|\xi(P)\|_{r}^{0} \leqq(2 m /(2-m))\left\|\nabla_{h} \xi(P)\right\|_{m}^{0}$.

LEMMA 3. If $\xi(P) \in H_{m, 0}^{1}\left(\Omega_{h}\right), m>2$, and $\Omega$ is strongly Lipschitz and bounded, then there exists a positive constant $C_{9}$ such that $\max _{P \in \Omega_{h}}|\xi(P)| \leqq C_{9}\left\|\nabla_{h} \xi(P)\right\|_{m}^{0}$ where $C_{9} \leqq 2 \sqrt{ } 2\left(C_{8}+3 m(\Omega) d\right) / m(\Omega)$.

LEMMA 4. If $f(P)$ is everywhere finite for $P \in \Omega_{h}$ and if $h^{2} \sum_{\Omega_{h}} f(P) \zeta(P)=0$ for all $\zeta(P) \in Q_{0}$, then, for all $P \in \Omega_{h}, f(P)=0$.

An immediate consequence of Lemma 4 is that a weak solution to the discrete problem in (3) exists iff a solution to the discrete problem in (1) exists for the same mesh width $h$. This lemma gives "numerical meaning" to the idea of a weak discrete solution. We introduce the weak solution idea only because we need some quantitative estimates in order to prove that solutions to (3), and hence (1), exist and to establish uniqueness and convergence criteria. This definition is made in "formal anology" with that used in partial differential equations.

We now turn our attention to the question of existence and uniqueness of a solution to (3). The case $m=2$ will be treated separately. We do not list explicitly all possible cases which can occur from our assumptions but only a representative sample to indicate the methods of proof. Some of our constraints on the constants of the problem, as given in the next theorem, come about as we are not able to prove all the results of the De Giorgi-Nash-Moser type; some general reasons for this were given in the Introduction. After the proof of the following theorem, we will give an example to which it may be applied.

THEOREM 1. We assume in all cases to be considered that Condition (F) holds.

(i) Let Conditions (A), (B), and (D) hold with $m>2$. If Conditions ( $\mathrm{C} \alpha)$ and $(\mathrm{E} \alpha)$ 
also hold with $C_{1}>C_{4} C_{8}^{1 / m} 2^{m^{\prime 2} / m}$, then (3) has a solution in $H_{m, 0}^{1}\left(\Omega_{h}\right)$. If Conditions $(\mathrm{C} \beta)$ and $(\mathrm{E} \beta)$ also hold and $\kappa>0$, then (3) has a solution in $H_{m, 0}^{1}\left(\Omega_{h}\right)$. If Condition $(\mathrm{E} \gamma)$ also holds, then (3) has a solution in $H_{m, 0}^{1}\left(\Omega_{h}\right)$.

(ii) If $m \in(4 / 3,2)$, if Conditions (A), (B), (D), ( $\mathrm{C} \alpha)$ and $(\mathrm{E} \alpha)$ hold and if $C_{1}>C_{4} C_{8}^{1 / m} 2^{m^{\prime 2} / m}$, then (3) has a solution in $H_{m, 0}^{1}\left(\Omega_{h}\right)$. Similar results hold for the other cases.

(iii) Let Conditions $\left(\mathrm{A}^{\prime}\right),\left(\mathrm{B}^{\prime}\right)$, and $\left(\mathrm{D}^{\prime}\right)$ hold with $m>2$. If Conditions $\left(\mathrm{C}^{\prime} \alpha\right)$ and (E' $\alpha)$ also hold with $C_{1}>C_{4} 3^{m^{\prime 2} / m} C_{8}^{1 / m}\left(C_{8}^{m^{\prime} / m}+1\right)$, then (3) has a solution in $H_{m, 0}^{1}\left(\Omega_{h}\right)$. Similar results hold for the other conditions.

(iv) If $m \in(4 / 3,2)$, if Conditions $\left(\mathrm{A}^{\prime}\right),\left(\mathrm{B}^{\prime}\right),\left(\mathrm{D}^{\prime}\right),\left(\mathrm{C}^{\prime} \alpha\right)$ and $\left(\mathrm{E}^{\prime} \alpha\right)$ hold and if $C_{1}>C_{4} 3^{m^{\prime 2} / m} C_{8}^{1 / m}\left(C_{8}^{m^{\prime} / m}+1\right)$, then (3) has a solution in $H_{m, 0}^{1}\left(\Omega_{h}\right)$. Similar results hold for the other conditions.

Proof. Case (a): $2<m$. We first assume that $(\mathrm{C} \alpha)$ and $(\mathrm{E} \alpha)$ hold with $C_{1}>C_{4} C_{8}^{1 / m} 2^{m^{\prime 2} / m}$.

Let $S_{1}=\left\{\xi(P): \xi(P) \in a_{0}, h^{2} \sum\left|\nabla_{h} \xi\right|^{m} \leqq J_{1}^{m}\right\}$ for some positive number $J_{1}$ which is independent of $h$. Let us "formally define" the quantity $\phi(\xi(\cdot) ; P)$ as a solution to the problem

$$
h^{2} \sum_{\Omega_{h^{\prime}}}\left\{a_{i}\left(P, \xi(P), \nabla_{h} \phi\right) \zeta_{x_{i}}+f\left(P, \xi, \nabla_{h} \xi\right) \zeta\right\}=0
$$

for all $\zeta \in a_{0}$ and with $\nabla_{h} \phi=\left(\phi_{x_{1}}(\xi(\cdot) ; P), \phi_{x},(\xi(\cdot) ; P)\right)$.

It follows from Condition (F) that to each $\xi(P) \in S_{1}$ there exists at least one $\phi(\xi(\cdot) ; P)$ and it follows from Condition (B) that to each $\xi(P) \in S_{1}$ there is at most one $\phi(\xi(\cdot) ; P)$.

Now, we determine those conditions which allow us to conclude that $\phi: S_{1} \rightarrow S_{1}$. In (5) let $\zeta=\phi$ and apply the conditions in (A) and (C) and Lemma 1 to get

$$
\begin{aligned}
C_{1} h^{2} \sum\left|\nabla_{h} \phi\right|^{m} & \leqq h^{2} \sum\left|f\left(P, \xi, \nabla_{h} \xi\right)\right| \cdot|\phi| \\
& \leqq C_{4} C_{8}^{1 / m} 2^{m^{\prime 2 / m}}\left\{J_{1}^{m^{\prime}}+\left(m_{h}\left(\Omega_{h}\right)\right)^{m^{\prime} / m}\right\}\left(h^{2} \sum\left|\nabla_{h} \phi\right|^{m}\right)^{1 / m} .
\end{aligned}
$$

Now we want the right-hand side of (6) to be $\leqq C_{1} J_{1}^{m-1}$, i.e. we want $J_{1}$ so that

$$
J_{1}^{m-1} \geqq C_{4} C_{8}^{1 / m} 2^{m^{\prime s / m}}\left(m_{h}\left(\Omega_{h}\right)\right)^{m^{\prime} / m} /\left(C_{1}-C_{4} C_{8}^{1 / m} 2^{m^{\prime 2 / m}}\right) .
$$

Hence, with $J_{1}$ satisfying (7), we have that $\phi: \S_{1} \rightarrow \S_{1}$.

We shall show that $\phi$ is a Hölder-continuous function of $\xi$ in the topology on $S_{1}$ induced by its defining norm. Let $\xi_{1}, \xi_{2} \in S_{1}$ with $\phi_{1}, \phi_{2}$ the associated solutions to (5). Then, if we set $\zeta=\phi_{1}-\phi_{2}$, we get: (the summation is over $P \in \Omega_{h}^{\prime}$ )

$$
\begin{aligned}
h^{2} \sum\left\{\left(a_{i}\left(P, \xi_{1}, \nabla_{h} \phi_{1}\right)-a_{i}\left(P, \xi_{1}, \nabla_{h} \phi_{2}\right)+a_{i}\left(P, \xi_{1}, \nabla_{h} \phi_{2}\right)-a_{i}\left(P, \xi_{2}, \nabla_{h} \phi_{2}\right)\right)\right. \\
\left.\cdot\left(\phi_{1}-\phi_{2}\right)_{x_{i}}+\left(f\left(P, \xi_{1}, \nabla_{h} \xi_{1}\right)-f\left(P, \xi_{2}, \nabla_{h} \xi_{2}\right)\right)\left(\phi_{1}-\phi_{2}\right)\right\}=0 .
\end{aligned}
$$

The Mean Value Theorem and our assumptions give

$$
\begin{aligned}
& h^{2} \sum \int_{0}^{1} a_{i, p_{i}}\left(P, \xi_{1},(1-t) \nabla_{h} \phi_{1}+t \nabla_{h} \phi_{2}\right) d t \cdot\left(\phi_{1}-\phi_{2}\right)_{x_{i}}\left(\phi_{1}-\phi_{2}\right)_{x_{i}} \\
& \leqq h^{2} \sum\left\{\left|\int_{0}^{1} a_{i, u}\left(P,(1-t) \xi_{1}+t \xi_{2}, \nabla_{h} \phi_{2}\right) d t \cdot\left(\xi_{1}-\xi_{2}\right) \cdot\left(\phi_{1}-\phi_{2}\right)_{x_{i}}\right|\right. \\
&\left.+\left(\left|\nabla_{h} \xi_{1}\right|^{2}+\left|\nabla_{h} \xi_{2}\right|^{2}+1\right)^{\bar{m} / 2} C_{7}\left(\left|\xi_{1}-\xi_{2}\right|+\left|\nabla_{h}\left(\xi_{1}-\xi_{2}\right)\right|\right)\left|\phi_{1}-\phi_{2}\right|\right\} .
\end{aligned}
$$


Now, for $m \geqq 2, \bar{m}=m-2, m^{\prime}=m-1$,

$$
\begin{aligned}
\left(\phi_{1}-\phi_{2}\right)_{x_{i}}\left(\phi_{1}-\phi_{2}\right)_{x_{i}} & \int_{0}^{1} a_{i, p_{j}}\left(P, \xi_{1},(1-t) \nabla_{h} \phi_{1}+t \nabla_{h} \phi_{2}\right) d t \\
& \geqq C_{2} \int_{0}^{1}\left\{\left|\nabla_{h}\left((1-t) \phi_{1}+t \phi_{2}\right)\right|^{2}+1\right\}^{\bar{m} / 2} d t\left|\nabla_{h}\left(\phi_{1}-\phi_{2}\right)\right|^{2} \\
& \geqq C_{2}^{\prime}\left|\nabla_{h}\left(\phi_{2}-\phi_{1}\right)\right|^{m} / m^{\prime},
\end{aligned}
$$

where $C_{2}^{\prime}=C_{2} / 2$ if $(m-2) / 2 \geqq 1$ and $C_{2}^{\prime}=2^{1 / m} C_{2} / 2^{2}$ if $0<(m-2) / 2<1$. Hence, for $m>2$,

$$
\begin{aligned}
& \left(C_{2}^{\prime} / m^{\prime}\right)\left(\left\|\nabla_{h}\left(\phi_{2}-\phi_{1}\right)\right\|_{m}^{0}\right)^{m} \\
& \leqq \\
& h^{2} \sum\left\{C_{6}\left(1+\left|\nabla_{h} \phi_{2}\right|^{2}\right)^{\bar{m} / 2}\left|\xi_{1}-\xi_{2}\right| \cdot\left|\nabla_{h}\left(\phi_{2}-\phi_{1}\right)\right|\right. \\
& \left.\quad+\left(1+\left|\nabla_{h} \xi_{1}\right|^{2}+\left|\nabla_{h} \xi_{2}\right|^{2}\right)^{\bar{m} / 2} C_{7}\left(\left|\xi_{1}-\xi_{2}\right|+\left|\nabla_{h}\left(\xi_{1}-\xi_{2}\right)\right|\right)\left|\phi_{1}-\phi_{2}\right|\right\} \\
& \leqq \\
& \quad C_{6}\left(h^{2} \sum\left(1+\left|\nabla_{h} \phi_{2}\right|^{2}\right)^{m / 2}\right)^{\bar{m} / m} C_{8}^{1 / m}\left\|\nabla_{h}\left(\xi_{1}-\xi_{2}\right)\right\|_{m}^{0}\left\|\nabla_{h}\left(\phi_{1}-\phi_{2}\right)\right\|_{m}^{0} \\
& \quad+\left(h^{2} \sum\left(1+\left|\nabla_{h} \xi_{1}\right|^{2}+\left|\nabla_{h} \xi_{2}\right|^{2}\right)^{m / 2}\right)^{\bar{m} / m} C_{8}^{(m+1) / m} C_{7}\left\|\nabla_{h}\left(\xi_{1}-\xi_{2}\right)\right\|_{m}^{0} \\
& \quad \cdot\left\|\nabla_{h}\left(\phi_{1}-\phi_{2}\right)\right\|_{m}^{0}+C_{8}^{1 / m} C_{7}\left\|\nabla_{h}\left(\xi_{1}-\xi_{2}\right)\right\|_{m}^{0}\left\|\nabla_{h}\left(\phi_{1}-\phi_{2}\right)\right\|_{m}^{0} .
\end{aligned}
$$

Therefore, for $m>2$,

$$
\begin{aligned}
& \left(C_{2}^{\prime} / m^{\prime}\right)\left(\left\|\nabla_{h}\left(\phi_{2}-\phi_{1}\right)\right\|_{m}^{0}\right)^{m^{\prime}} \\
& \leqq\left\{C_{8}^{1 / m} C_{6}\left(2^{\bar{m} / 2}\left(J_{1}^{m}+m_{h}\left(\Omega_{h}\right)\right)\right)^{\bar{m} / m}\right. \\
& \left.\quad+C_{8}^{(m+1) / m} C_{7}\left(3^{m^{\prime}}\left(m_{h}\left(\Omega_{h}\right)+2 J_{1}^{m}\right)\right)^{\bar{m} / m}+C_{8}^{1 / m} C_{7}\right\}\left\|\nabla_{h}\left(\xi_{1}-\xi_{2}\right)\right\|_{m}^{0} .
\end{aligned}
$$

This is the definition of a Hölder-continuous function of $\xi \in S_{1}$ with respect to the norm on $S_{1}$. The Hölder exponent is independent of the mesh size $h$ if $J_{1}$ has this property.

The existence of an element $U(P) \in S_{1}$ satisfying the equation in (3) is now an immediate consequence of the Brouwer Fixed Point Theorem.

Now, consider that the Conditions $(\mathrm{C} \beta)$ and $(\mathrm{E} \beta)$ hold. The only part of the above proof which is in need of analysis is the determination of $J_{1}$. Here we have, using Young's Inequality,

$$
\begin{array}{r}
C_{1} h^{2} \sum\left|\nabla_{h} \phi\right|^{m} \leqq h^{2} \sum\left|f\left(P, \xi, \nabla_{h} \xi\right)\right| \cdot|\phi| \leqq h^{2} \sum|\phi| \cdot\left(1+\left|\nabla_{h} \xi\right|^{2}\right)^{(m-1-\kappa) / 2} \\
\leqq C_{8}^{1 / m} C_{7}^{\prime}\left\|\nabla_{h} \phi\right\|_{m}^{0}\left\{m_{h}\left(\Omega_{h}\right)+\epsilon^{m /\left(m-\kappa m / m^{\prime}\right)}\left(m-\kappa m / m^{\prime}\right)\left(\left\|\nabla_{h} \xi\right\|_{m}^{0}\right)^{m} / m\right. \\
\left.+\kappa m_{h}\left(\Omega_{h}\right) / m^{\prime} \epsilon^{m^{\prime} / k}\right\}^{m^{\prime} / m}
\end{array}
$$

where $C_{7}^{\prime}=C_{7} \cdot 2^{m-1-\kappa-m^{\prime} / m}$ if $m-\kappa m / m^{\prime} \geqq 1$ and $C_{7}^{\prime}=C_{7}$ otherwise, and $\epsilon>0$. Therefore, for $\tilde{\epsilon}=\epsilon^{m /\left(m-\kappa m / m^{\prime}\right)}$, we choose $\tilde{\epsilon}$ so that (for example), $C_{8}^{1 / m} C_{7}^{\prime} \tilde{\epsilon}^{m^{\prime} / m}\left(m-\kappa m / m^{\prime}\right)=C_{1} / 2$. Then pick $J_{1}$ so large that

$$
C_{8}^{1 / m} C_{7}^{\prime}\left(m_{h}\left(\Omega_{h}\right)\right)^{m^{\prime} / m}+\left\{\kappa m_{h}\left(\Omega_{h}\right) / m^{\prime} \epsilon^{m^{\prime} \times}\right\}^{m^{\prime} / m} \leqq C_{1} J_{1}^{m^{\prime}} / 2 .
$$

We now proceed as in the earlier situation, since the left-hand side of the above inequality is known.

Now consider the case that $(\mathrm{E} \gamma)$ holds. Using Lemma 3 we have that 


$$
C_{1} h^{2} \sum\left|\nabla_{h} \phi\right|^{m} \leqq \max _{P \in \Omega_{h}}|\phi| \cdot h^{2} \sum|f| \leqq C_{9}\left(h^{2} \sum\left|\nabla_{h} \phi\right|^{m}\right)^{1 / m} \cdot C_{7}
$$

and hence we choose $J_{1}$ so that $C_{9} C_{7} \leqq C_{1} J_{1}^{m^{\prime}}$.

Case (b): $4 / 3 \leqq m<2$. Assume that ( $\mathrm{E} \alpha)$ and $(\mathrm{C} \alpha)$ hold. We prove the continuity of $\phi(\xi(\cdot) ; P)$ over $S_{1}$.

We obtain, using the Hölder Inequality,

$$
\begin{aligned}
& h^{2} \sum\left(\phi_{1}-\phi_{2}\right)_{x_{i}}\left(\phi_{1}-\phi_{2}\right)_{x_{i}} \int_{0}^{1} a_{i, p_{i}}\left(P, \xi_{1},(1-t) \nabla_{h} \phi_{1}+t \nabla_{h} \phi_{2}\right) d t \\
& \geqq C_{2} h^{2} \sum\left\{\int_{0}^{1}\left\{1+\left|\nabla_{h}\left\{(1-t) \phi_{1}+t \phi_{2}\right\}\right|^{2}\right\}^{\bar{m} / 2} d t\left|\nabla_{h}\left(\phi_{1}-\phi_{2}\right)\right|^{2}\right\} \\
& \geqq C_{2}\left(h^{2} \sum\left(\int_{0}^{1}\left\{1+\left|\nabla_{h}\left\{(1-t) \phi_{1}+t \phi_{2}\right\}\right|^{2}\right\}^{\bar{m} / 2} d t\right)^{m / \bar{m}}\right)^{\bar{m} / m}\left(|| \nabla_{h}\left(\phi_{1}-\phi_{2}\right)||_{m}^{0}\right)^{2} .
\end{aligned}
$$

Therefore,

$$
\begin{gathered}
\boldsymbol{C}_{2}\left(|| \nabla_{h}\left(\phi_{1}-\phi_{2}\right)||_{m}^{0}\right)^{2} \leqq\left(h^{2} \sum\left(\int_{0}^{1}\left\{1+\left|\nabla_{h}\left\{(1-t) \phi_{1}+t \phi_{2}\right\}\right|^{2}\right\}^{\bar{m} / 2} d t\right)^{m / \bar{m}}\right)^{-\bar{m} / m} \\
\cdot\left\{\left(C_{6}+C_{7}\right) h^{2} \sum\left|\xi_{1}-\xi_{2}\right| \cdot\left|\nabla_{h}\left(\phi_{1}-\phi_{2}\right)\right|+C_{7} h^{2} \sum\left|\nabla_{h}\left(\xi_{1}-\xi_{2}\right)\right| \cdot\left|\phi_{1}-\phi_{2}\right|\right\} .
\end{gathered}
$$

Now

$$
\left(\int_{0}^{1}\left\{1+\left|\nabla_{h}\left\{(1-t) \phi_{1}+t \phi_{2}\right\}\right|^{2}\right\}^{\bar{m} / 2} d t\right)^{m / \bar{m}} \leqq\left(1+\left|\nabla_{h} \phi_{1}\right|^{2}+\left|\nabla_{h} \phi_{2}\right|^{2}\right)^{-m / 2}
$$

and hence, using the Hölder Inequality and Lemma 2,

$$
\begin{aligned}
C_{2}( & \left.\left\|\nabla_{h}\left(\phi_{1}-\phi_{2}\right)\right\|_{m}^{0}\right)^{2} \leqq\left\{2^{(2+m) / 2} J_{1}^{m}+m_{h}\left(\Omega_{h}\right)\right\}^{-\bar{m} / m} \\
& \cdot\left\{\left(C_{6}+C_{7}\right) h^{2} \sum\left|\xi_{1}-\xi_{2}\right| \cdot\left|\nabla_{h}\left(\phi_{1}-\phi_{2}\right)\right|+C_{7} h^{2} \sum\left|\nabla_{h}\left(\xi_{1}-\xi_{2}\right)\right| \cdot\left|\phi_{1}-\phi_{2}\right|\right\} \\
\leqq & \left\{2^{(2+m) / 2} J_{1}^{m}+m_{h}\left(\Omega_{h}\right)\right\}^{\bar{m} / m}\left\{\left(C_{6}+2 C_{7}\right)(-2 m / \bar{m})^{m^{\prime} / m}\right\} \cdot\left\|\nabla_{h}\left(\phi_{1}-\phi_{2}\right)\right\|_{m}^{0} \\
& \cdot\left\|\nabla_{h}\left(\xi_{1}-\xi_{2}\right)\right\|_{m}^{0} .
\end{aligned}
$$

Note that this shows that the function $\phi(\xi(\cdot) ; P)$ is a Lipschitz function on $S_{1}$.

The remainder of the proof proceeds exactly as in Case (a), even to the use of Lemma 2 in establishing the mapping property of $\phi$, i.e. for the computation of $J_{1}$.

Now, we consider the mapping property when $\left(\mathrm{A}^{\prime}\right),\left(\mathrm{B}^{\prime}\right),\left(\mathrm{C}^{\prime} \alpha\right),\left(\mathrm{D}^{\prime}\right)$ and $\left(\mathrm{E}^{\prime} \alpha\right)$ hold for $m>4 / 3$. As in (6), we have

$$
\begin{aligned}
C_{1} h^{2} \sum\left|\nabla_{h} \phi\right|^{m} & \leqq h^{2} \sum\left|f\left(P, \xi, \nabla_{h} \xi\right)\right| \cdot|\phi| \\
& \leqq C_{4} C_{8}^{1 / m}\left\|\nabla_{h} \phi\right\|_{m}^{0}\left(h^{2} \sum\left(1+\xi^{2}+\left|\nabla_{h} \xi\right|^{2}\right)^{m / 2}\right)^{m^{\prime} / m} ;
\end{aligned}
$$

that is

$$
C_{1}\left(\left\|\nabla_{h} \phi\right\|_{m}^{0}\right)^{m^{\prime}} \leqq C_{4} C_{8}^{1 / m} 3^{m^{\prime 2} / m}\left\{\left(m_{h}\left(\Omega_{h}\right)\right)^{m^{\prime} / m}+\left(C_{8}^{m^{\prime} / m}+1\right)\left(\left\|\nabla_{h} \xi\right\|_{m}^{0}\right)^{m^{\prime}}\right\} .
$$

Hence, we must require that

$$
C_{1}>C_{4} 3^{m^{\prime 2 / m}} C_{8}^{1 / m}\left(C_{8}^{m^{\prime} / m}+1\right)
$$

in order to establish the mapping property. 
The Hölder constant now comes from the relation

$$
\begin{aligned}
\left(C_{2}^{\prime} / m^{\prime}\right)\left(\left\|\nabla_{h}\left(\phi_{2}-\phi_{1}\right)\right\|_{m}^{0}\right)^{m} & \\
\leqq & \left\{C_{6}\left(h^{2} \sum\left(1+\left|\xi_{1}\right|^{2}+\left|\xi_{2}\right|^{2}+\left|\nabla_{h} \phi_{2}\right|^{2}\right)^{m / 2}\right)^{\bar{m} / m} C_{8}^{1 / m}+C_{8}^{j / m} C_{7}\right. \\
& \left.\quad+\left(h^{2} \sum\left(1+\left|\xi_{1}\right|^{2}+\left|\xi_{2}\right|^{2}+\left|\nabla_{h} \xi_{1}\right|^{2}+\left|\nabla_{h} \xi_{2}\right|^{2}\right)^{m / 2}\right)^{\bar{m} / m} C_{8}^{(m+1) / m} C_{7}\right\} \\
& \cdot\left\|\nabla_{h}\left(\phi_{1}-\phi_{2}\right)\right\|_{m}^{0} \cdot\left\|\nabla_{h}\left(\xi_{1}-\xi_{2}\right)\right\|_{m}^{0} .
\end{aligned}
$$

Now, use the mapping property to complete the bound on the Hölder constant.

The case that $4 / 3 \leqq m<2$ yields a Lipschitz constant computed from the relation, assuming that the primed conditions hold,

$$
\begin{aligned}
& \left\|\nabla_{h}\left(\phi_{1}-\phi_{2}\right)\right\|_{m}^{0} \\
& \quad \leqq\left\{\left(2^{(2+m) / 2} J_{1}^{m}+m_{h}\left(\Omega_{h}\right)\right)^{-\bar{m} / m} C_{2}\right\}\left\{\left(C_{6}+2 C_{7}\right)(2 m / \bar{m})^{m^{\prime} / m}\right\}\left\|\nabla_{h}\left(\xi_{1}-\xi_{2}\right)\right\|_{m}^{0} .
\end{aligned}
$$

Remark 1. If we assume that the inhomogeneous term $f(P, \xi, \nabla \xi)$ satisfies a condition of the type,

$$
\sum\left\{\left|f\left(P, \xi, \nabla_{h} \xi\right)\right|+\nu_{i}\left|\partial f / \partial \xi_{x_{i}}\right|\right\}+\nu_{3}|\partial f / \partial \xi| \leqq C_{7},
$$

where $\nu_{1}, \nu_{2}$ and $\nu_{3}$ are positive constants, then our mapping property is always established for $m>2$ when we pick $C_{7} m_{h}\left(\Omega_{h}\right) \leqq C_{1} J_{1}^{m-2}$ and when we set $\nu_{i}=0$ for $j=1,2,3$, in the above. If $\nu_{i} \in\{0,1\}$ and $\partial a_{i} / \partial \xi=0$ over $S_{1}$, then the continuity condition takes the simpler form, setting

$$
\begin{aligned}
{\left[\nu_{1}, \nu_{2}\right] } & =\max \left\{\nu_{1}, \nu_{2}\right\}, \\
\left\|\nabla_{h}\left(\phi_{1}-\phi_{2}\right)\right\|_{m}^{m} & \leqq C_{7} h^{2} \sum\left\{\left[\nu_{1}, \nu_{2}\right]\left|\nabla_{h}\left(\xi_{1}-\xi_{2}\right)\right|+\nu_{3}\left|\xi_{1}-\xi_{2}\right|\right\}\left|\phi_{1}-\phi_{2}\right| .
\end{aligned}
$$

This case occurs if, for example, $f=\sin \left\{\xi_{x_{1}}+\xi_{x_{1}}+\xi\right\}$.

We will illustrate some of the ideas of the last result in the following example.

Example. Let $a_{i}\left(\nabla_{h} U\right)=\left(1+\left|\nabla_{h} U\right|^{2} / \bar{m}\right)^{\bar{m} / 2} U_{x_{i}}$ for $m>2$. Then, $a_{i}\left(\nabla_{h} \phi\right)=$ $(\bar{m} / m) \partial\left(1+\left|\nabla_{h} \phi\right|^{2} / \bar{m}\right)^{m / 2} / \partial \phi_{x_{i}}$ and hence Condition $(\mathrm{F})$ is satisfied. Also, we have that $C_{1}=\bar{m}^{-\bar{m} / 2}=C_{2}$ if $m \geqq 3, C_{1}=C_{2}=1$ if $m \in(2,3], C_{3}=3 / \bar{m}$ if $m \in(2,3)$, $C_{3}=1+2 \bar{m}^{2}$ if $m \geqq 3, C_{5}=\bar{m}^{1 / 2}$ if $m \geqq 3$, and $C_{5}=\bar{m}^{\left(1-m^{\prime}\right) / 2}$ if $m \in(2,3)$. Suppose we consider the problem of finding a mesh function $\phi(\xi(\cdot) ; P) \in Q_{0}\left(\Omega_{h}\right)$ such that for every $\zeta(P) \in a_{0}\left(\Omega_{h}\right)$ we have

$$
h^{2} \sum\left\{\left(1+\left|\nabla_{h} \phi\right|^{2} / \bar{m}\right)^{\bar{m} / 2} \phi_{x_{i}} \zeta_{x_{i}}+\left(1+\left|\nabla_{h} \xi\right|^{2}+\sin ^{2} \xi\right)^{m^{\prime} / 2} \zeta\right\}=0
$$

where $\Omega_{h}$ has not been specified yet. Note that $C_{4}=2$. If we can find a mesh function $\psi \in Q_{0}\left(\Omega_{h}\right)$ such that

$$
I(\psi)=\min _{V \in a_{0}} I(V)
$$

where

$$
I(V)=h^{2} \sum\left\{(\bar{m} / m)\left(1+\left|\nabla_{h} V\right|^{2} / \bar{m}\right)^{m / 2}+\left(1+\left|\nabla_{h} \xi\right|^{2}+\sin ^{2} \xi\right)^{m^{\prime} / 2} V\right\},
$$

then this mesh function $\psi$ is what we will call $\phi$. If we require that $C_{1}^{m}>C_{4}^{m} C_{8} 2^{m^{\prime} m^{\prime}}$, i.e. $1>2^{m+m^{\prime} m^{\prime+1}} \max \left(d_{x_{1}}^{2}, d_{x_{2}}^{2}\right)$ for $m \in(2,3)$ and $>\bar{m}^{\bar{m} m / 2} 2^{m+m^{\prime} m^{\prime}+1} \max \left(d_{x_{1}}^{2}, d_{x_{2}}^{2}\right)$ for $m \geqq 3$, then a constant $J_{1}$ exists and the set $S_{1}$ is meaningful. In fact, we may take 


$$
\begin{aligned}
& J_{1}^{m^{\prime} / m}=2^{2 m+m^{\prime} m^{\prime}}\left(\max \left(d_{x_{1}}^{2}, d_{x_{2}}^{2}\right)\right)^{m} /\left(1-2^{m+n^{\prime} m^{\prime}+1} \max \left(d_{x_{1}}^{2}, d_{x_{2}}^{2}\right)\right), \quad m \in(2,3], \\
& =2^{2 m+m^{\prime} m^{\prime}}\left(\max \left(d_{x_{1}}^{2}, d_{x_{2}}^{2}\right)\right)^{m} /\left(1-\bar{m}^{\bar{m} m / 2} 2^{m+m^{\prime} m^{\prime}+1} \max \left(d_{x_{1}}^{2}, d_{x_{2}}^{2}\right)\right), \quad m \geqq 3 .
\end{aligned}
$$

Then, for $V \in Q_{0}\left(\Omega_{h}\right), \xi \in \delta_{1}$ and $\epsilon>0$, we have that

$$
I(V) \geqq\left(1 / m m^{\bar{m}}-\epsilon / m\right)\left\|\nabla_{h} V\right\|_{m}^{m}-\left(2^{\bar{m}}+m 2^{(\bar{m} m+1) / m^{\prime}} J_{1}^{m} / \epsilon m^{\prime}\right)\left(\max \left(d_{x_{1}}^{2}, d_{x_{2}}^{2}\right)\right)^{m / m^{\prime}} .
$$

Now, choose $\epsilon$ so that $1 / m \bar{m}^{\bar{m}}-\epsilon / m>0$ and we have that $I(V)$ is bounded from below on $a_{0}\left(\Omega_{h}\right)$. Since $I(V)$ is clearly continuous, we have that an $\psi$ exists. Now call $\phi(\xi(\cdot) ; P)$ this mesh function $\psi$.

As an almost immediate consequence of Theorem 1, we have the next result.

COROLlary 1. If $4 / 3 \leqq m<2$ and if

$$
\left\{2^{(2+m) / 2} J_{1}^{m}+m_{h}\left(\Omega_{h}\right)\right\}^{(2-m) / m}\left\{\left(C_{6}+2 C_{7}\right)(2 m /(2-m))^{(m-1) / m}\right\} \leqq C_{2},
$$

whenever conditions $(\mathrm{A}),(\mathrm{B}),(\mathrm{D})$, and $(\mathrm{C} \alpha),(\mathrm{E} \alpha)$, or $(\mathrm{C} \beta),(\mathrm{E} \beta)$, or $(\mathrm{E} \gamma)$ hold, or if

$$
\left\{2^{(2+m) / 2} J_{1}^{m}+m_{h}\left(\Omega_{h}\right)\right\}^{-\bar{m} / m}\left\{\left(C_{6}+2 C_{7}\right)(-2 m / \bar{m})^{m^{\prime} / m}\right\}<C_{2},
$$

whenever Conditions $\left(\mathrm{A}^{\prime}\right),\left(\mathrm{B}^{\prime}\right),\left(\mathrm{D}^{\prime}\right)$, and $\left(\mathrm{C}^{\prime} \alpha\right),\left(\mathrm{E}^{\prime} \alpha\right)$, or $\left(\mathrm{C}^{\prime} \beta\right),\left(\mathrm{E}^{\prime} \beta\right)$ hold, then $\phi(\xi(\cdot) ; P)$ is a Lipschitz function on $\mathrm{S}_{1}$ and has one and only one fixed-point, i.e. one and only one solution to (3) exists.

We shall now consider the case that $m=2$ in establishing criteria for the constructive existence and uniqueness.

THEOREM 2. If conditions $\left(\mathrm{A}^{\prime}\right),\left(\mathrm{B}^{\prime}\right),\left(\mathrm{C}^{\prime} \alpha\right),\left(\mathrm{E}^{\prime} \alpha\right)$ and $\left(\mathrm{D}^{\prime}\right)$ are satisfied with $m=2$, then (3) has a unique solution in $Q_{0}\left(\Omega_{h}\right)$ if there exists a positive constant $\mu$ such that

$$
1-\mu C_{2}+\mu\left\{\left(C_{6}+C_{7}\right) C_{8}^{1 / 2}+C_{7} C_{8}\right\}<1 ;
$$

in this case the solution is completely constructible. Note that this condition is satisfied if $\left(\mathrm{E}_{\gamma}\right)$ holds with $\partial a_{i} / \partial V \equiv 0$.

Proof. Let $\delta_{2}=\left\{\xi(P): \xi(P) \in a_{0}\left(\Omega_{h}\right),\left\|\nabla_{h} \xi\right\|_{m} \leqq J_{1}\right\}$ and define the function $\phi(\xi(\cdot) ; P)$ as the solution to the problem, for $\mu$ a parameter,

$$
h^{2} \sum\left\{\phi_{x_{i}} \zeta_{x_{i}}-\xi_{x_{i}} \zeta_{x_{i}}+\mu a_{i}\left(P, \xi, \nabla_{h} \xi\right) \zeta_{x_{i}}+\mu f\left(P, \xi, \nabla_{h} \xi\right) \zeta\right\}=0 .
$$

Remark 2. We observe that in all cases considered on $m$, if $\partial a_{i} / \partial V=0$ and $f$ depends on $P$ alone, then a solution to (3) exists and it must be unique.

It is natural at this point, especially after our last result, to determine if we may remove the very confining Condition $(F)$. We shall state and prove a theorem on a sufficient condition for the removal of this condition for the case $m>2$. The case $m \in[4 / 3,2)$ is treated in a similar manner and the modifications necessary will be read off from what we give.

THEOREM 3. If the hypotheses of Theorem 1(iii) hold, with the exception of Condition (F), and if a positive constant $\mu$ exists such that $\mu C_{2}-2^{m^{\prime}} m^{\prime} \geqq 0$ and

$$
\mu\left(C_{5}+C_{4} C_{8}^{1 / m}\right) 3^{m^{\prime} m^{\prime} / m}\left(1+C_{8}\right)^{m^{\prime} / m}<1,
$$

then (3) has a solution in $H_{m, 0}^{1}\left(\Omega_{h}\right)$.

Proof. We need only prove that $\phi(\xi(\cdot) ; P)$ exists for all $\xi(P) \in \mathrm{S}_{1}$ as this was the only place Condition (F) was used. Let $\psi(\nu(\cdot) ; P)$, for $\nu(P) \in S_{1}$ and $\mu$ a parameter to be determined, be a solution to 


$$
\begin{aligned}
& h^{2} \sum\left\{\left\{\left(1+\left|\nabla_{h} \psi\right|^{2}\right)^{\bar{m} / 2} \psi_{x_{i}}+\mu a_{i}(P, \xi, 0)\right.\right. \\
& \left.\left.\quad+\mu \tilde{a}_{i, p_{i}} \psi_{x_{i}}-\left(1+\left|\nabla_{h} \nu\right|^{2}\right)^{\bar{m} / 2} \psi_{x_{i}}\right\} \zeta_{x_{i}}+\mu f\left(P, \xi, \nabla_{h} \xi\right) \zeta\right\}=0,
\end{aligned}
$$

where

$$
\tilde{a}_{i, p_{i}}=\int_{0}^{1} a_{i, p_{i}}\left(P, \xi, t \nabla_{h} \nu\right) d t
$$

Clearly, $\psi(\nu(\cdot) ; P)$ exists as

$$
I(\psi)=\min _{V \in s_{1}} I(V)
$$

where

$$
\begin{gathered}
I(V)=h^{2} \sum\left\{\left(1+\left|\nabla_{h} V\right|^{2}\right)^{m / 2} / m+\mu a_{i}(P, \xi, 0) V_{x_{i}}+\mu \tilde{a}_{i, p_{i}} V_{x_{i}} V_{x_{j}}\right. \\
\left.-\left(1+\left|\nabla_{h} \nu\right|^{2}\right)^{\bar{m} / 2} V_{x_{i}}^{2}+\mu f\left(P, \xi, \nabla_{h} \xi\right) V\right\} .
\end{gathered}
$$

Now, we show that $\psi: S_{1} \rightarrow \delta_{1}$. As in Theorem 1, we have that

$$
\left\|\nabla_{h} \psi\right\|_{m}^{m^{\prime}} \leqq \mu\left(C_{5}+C_{4} C_{8}^{1 / m}\right) 3^{m^{\prime} m^{\prime} / m}\left\{\left(m_{h}\left(\Omega_{h}\right)\right)^{m^{\prime} / m}+\left(1+C_{8}\right)^{m^{\prime} / m} J_{1}^{m^{\prime}}\right\} .
$$

Now, pick $J_{1}^{m^{\prime}}=\max \left\{A_{1}, A_{2}\right\}$ where

$$
\begin{aligned}
& A_{1}=\mu\left(C_{5}+C_{4} C_{8}^{1 / m}\right) 3^{m^{\prime} m^{\prime} / m} B \div\left(1-\mu\left(C_{5}+C_{4} C_{8}^{1 / m}\right) 3^{m^{\prime} m^{\prime} / m}\left(1+C_{8}\right)^{m^{\prime} / m}\right), \\
& A_{2}=C_{4} C_{8}^{1 / m} 2^{m^{\prime} m^{\prime} / m} B \div\left(C_{1}-C_{4} C_{8}^{1 / m} 2^{m^{\prime} m^{\prime} / m}\right)
\end{aligned}
$$

and

$$
B=\left(m_{h}\left(\Omega_{h}\right)\right)^{m^{\prime} / m} ;
$$

note that $A_{2}$ comes from (7) when the primed conditions are used. Now we have that $\psi: S_{1} \rightarrow \delta_{1}$.

Now, observe that $\left\|\nu_{n}-\nu\right\|_{m}^{1} \rightarrow 0$ iff $\nu_{n}-\nu_{0} \rightarrow 0$ for each $P \in \Omega_{h}$. Hence $\psi$ is a continuous, in $H_{m, 0}^{1}\left(\Omega_{h}\right)$ norm, function of $\nu(P) \in S_{1}$. By Brouwer's Fixed Point Theorem, at least one fixed point of $\psi$ exists on $\mathrm{S}_{1}$; we call one of these $\phi(\xi(\cdot) ; P)$.

Now, we shall obtain "interior estimates" for powers of second-order difference quotients, i.e. we shall show that for any subregion $D$ of $\Omega$ such that $\bar{D} \subset \Omega$, we have that $\left|\nabla_{h} U_{x_{0}}\right|, s=1$ or 2 , is in $l_{p}\left(D_{h}\right)$ for some $p>0$ and for all $h$. These estimates will be used when we prove the convergence of the solutions of the difference equation to a solution of the differential equation. In a remark at the end of the proof of the next theorem, we will explain the generality of parts (d) and (e) in the statement of the next theorem.

THEOREM 4. (a) If $U(P)$ is a solution to (3), if $h^{2} \sum\left|\nabla_{h} U\right|^{m}$ is bounded-independent of $h$-over its domain of definition, if $m>2$, and if conditions $(\mathrm{B}),(\mathrm{C} \alpha)$ and (D) hold, then there exists a positive constant $J_{4}$ which is independent of $h$ such that

$$
h^{2} \sum \eta^{2}\left(1+\left|\nabla_{h} U\right|^{2}\right)^{(m-2) / 2}\left|\nabla_{h} U_{x_{1}}\right|^{2} \leqq J_{4},
$$

where $\eta(P)$ is a smooth mesh function with support over compact mesh regions of $\Omega_{h}$, and $J_{4}$ is given in (10), and $U_{x_{0}}=U_{x_{1}}$ or $U_{x_{2}}$.

(b) If $U(P)$ is a solution of (3) with $m=2$ and if the corresponding hypotheses of (2) are satisfied, then $h^{2} \sum \eta^{2}\left|\nabla_{h} U_{x}\right|^{2} \leqq J_{5}$ with $J_{6}$ given in (11). 
(c) If $U(P)$ is a solution to (3) with $m \in[4 / 3,2]$ and the appropriate hypotheses hold as in (a), then $h^{2} \sum \eta^{m}\left|\nabla_{h} U_{x}\right|^{m} \leqq J_{6}^{m / 2}$ where $J_{6}$ is given in (12).

(d) If $m \geqq 2$ and $\xi(P) \in S_{3}$ where, for some positive number $J_{7}, S_{3}=\left\{\xi(P): \xi(P) \in S_{1}\right.$ and $\left\|\nabla_{h} \xi\right\|_{2(m-1-\kappa)}^{0} \leqq J_{7}$ for $\left.\kappa \geqq 0\right\}$, if Conditions (B), (C), and (D) are satisfied, and if $\phi(\xi(\cdot) ; P) \in H_{m, 0}^{1}\left(\Omega_{h}\right)$ for each $\xi \in S_{3}$, then there exists a positive constant $J_{8}$ which depends on $J_{7}$ and other quantities such that

$$
h^{2} \sum \eta^{2}\left(1+\left|\nabla_{h} \phi\right|^{2}\right)^{(m-2) / 2}\left|\nabla_{h} \phi_{x_{1}}\right|^{2} \leqq J_{8} .
$$

The number $J_{8}$ is estimated in (13).

(e) If $m \in[4 / 3,2]$, if $\xi(P) \in S_{4}$ where, for some positive number $J_{9}$,

$S_{4}=\left\{\xi(P): \xi(P) \in S_{1}\right.$ and $\left.\max \left[\left\|\nabla_{h} \xi\right\|_{2(m-1-x)},\left\|\nabla_{h} \xi\right\|_{m /(m-1)}\right] \leqq J_{9}, \kappa>0\right\}$,

if Conditions (B), (C), and (D) are satisfied, and if $\phi(\xi(\cdot) ; P) \in H_{m}^{1}\left(\Omega_{h}\right)$, then a positive constant $J_{10}$ exists such that

$$
h^{2} \sum \eta^{m}\left|\nabla_{h} \phi_{x}\right|^{m} \leqq J_{10} .
$$

Proof. (a) Let $\zeta=\mu_{x}$, for $s=1$ or 2 , and $\mu_{x}(P) \in a_{0}\left(\Omega_{h}\right)$. Then, using Gauss' Theorem, we obtain

$$
\begin{aligned}
h^{2} \sum a_{i}\left(P, U(P), \nabla_{h} U(P)\right) \zeta_{x_{i}} & =-h^{2} \sum\left(a_{i}\left(P, U, \nabla_{h} U\right)\right)_{x_{i}} \mu_{x_{i}} \\
& =-h^{2} \sum\left\{\tilde{a}_{i, p_{k}} U_{x_{k} x_{i}}+\tilde{a}_{i, u} U_{x_{i}}+\tilde{a}_{i, x_{i}}\right\} \mu_{x_{i}},
\end{aligned}
$$

where

$$
\begin{aligned}
& \tilde{a}_{i, p_{k}}=\int_{0}^{1} a_{i, p h}\left(P, U, \nabla_{h} \tilde{U}\right) d t, \quad \tilde{U}=(1-t) U\left(P-h_{s}\right)+t U(P), \\
& \tilde{a}_{i, u}=\int_{0}^{1} a_{i, u}\left(P, \tilde{U}, \nabla_{h} U\left(P-h_{s}\right)\right) d t, \quad P-h_{1}=\left(x_{p}-h, y_{p}\right), \\
& \tilde{a}_{i, x_{s}}=\int_{0}^{1} a_{i, x_{s}}\left(\tilde{P}, U\left(P-h_{s}\right), \nabla_{h} U\left(P-h_{s}\right)\right) d t, \quad \tilde{P}=(1-t)\left(P-h_{s}\right)+t P .
\end{aligned}
$$

Substitution of (9) into (3) gives

$$
h^{2} \sum\left\{\left\{\tilde{a}_{i, p_{k}} U_{x_{k} x_{t}}+\tilde{a}_{i, u} U_{x_{t}}+\tilde{a}_{i, x_{\ell}}\right\} \mu_{x_{i}}-f\left(P, U, \nabla_{h} U\right) \mu_{x_{\iota}}\right\}=0 .
$$

Let $\eta(P)$ be a nonnegative mesh function such that the closure, relative to our neighborhood system, of its support is contained in a mesh region $D_{h}$ such that $\bar{D}_{k} \subset \Omega_{h}$. Let $\mu(P)=\eta^{2}(P) U_{x_{s}}(P)$. Using [8, p. 10] and Conditions (B), (D), (C $\left.\alpha\right)$, we obtain the estimate

$C_{2} h^{2} \sum \eta^{2}\left(1+\left|\nabla_{h} U\right|^{2}\right)^{\bar{m} / 2}\left|\nabla_{h} U_{x_{i}}\right|^{2}$

$$
\begin{aligned}
\leqq h^{2} \sum & \left\{\left(12 C_{3}\left|\nabla_{h} \eta\right| \eta+\eta^{2} C_{6}\right)\left(1+\left|\nabla_{h} U\right|^{2}\right)^{\bar{m} / 2}\left|\nabla_{h} U_{x_{s}}\right| \cdot\left|U_{x_{s}}\right|\right. \\
& \cdot\left(\eta^{2} C_{5}^{\prime}+4\left|\nabla_{h} \eta\right|\left(\eta\left|U_{x_{s}}\right|^{2} C_{6}+\eta\left|U_{x_{s}}\right| C_{5}^{\prime}\right)\right)\left(1+\left|\nabla_{h} U\right|^{2}\right)^{\bar{m} / 2} \\
& \left.+C_{4}\left(1+\left|\nabla_{h} U\right|^{2}\right)^{m^{\prime} / 2}\left(\eta^{2}\left|\nabla_{h} U_{x_{s}}\right|+\eta 2\left|\nabla_{h} \eta\right| \cdot\left|U_{x_{s}}\right|\right)\right\} .
\end{aligned}
$$

Using the Cauchy Inequality, for $\epsilon_{i}>0(i=1, \cdots, 4)$ and so that $12 C_{3} \epsilon_{1}+C_{6} \epsilon_{2}+$ $C_{5}^{\prime} \epsilon_{3}+C_{4} \epsilon_{4}=C_{2}$ with $\epsilon_{1}=C_{2} / 48 C_{3}, \epsilon_{2}=C_{2} / 4 C_{6}, \epsilon_{3}=C_{2} / 4 C_{5}^{\prime}$, and $\epsilon_{4}=C_{2} / 4 C_{4}$, then we obtain the bound 


$$
\begin{aligned}
\frac{1}{2} C_{2} h^{2} \sum & \eta^{2}\left(1+\left|\nabla_{h} U\right|^{2}\right)^{\bar{m} / 2}\left|\nabla_{h} U_{x}\right|^{2} \\
\leqq & 2^{m^{\prime}} h^{2} \sum\left\{2 \eta^{2} C_{4}^{2} / C_{2}+\left(288 C_{3}^{2}+2 C_{6}^{2}+2 C_{5}^{\prime}\right)\left|\nabla_{h} \eta\right|^{2} / C_{2}\right. \\
& \left.\quad+2 \eta\left|\nabla_{h} \eta\right|\left(C_{6}+C_{4}\right)\right\}\left(1+\left|\nabla_{h} U\right|^{m}\right) \\
& \quad+\max \left\{1,2^{\bar{m}}\right\} h^{2} \sum 2\left|\nabla_{h} \eta\right| \eta C_{5}^{\prime}\left(1+\left|\nabla_{h} U\right|^{m^{\prime}}\right) .
\end{aligned}
$$

If we now choose $|\eta(P)|$ and $\left|\nabla_{h} \eta(P)\right|$ to be bounded over $\bar{\Omega}_{h}$, then we have that $\eta\left(1+\left|\nabla_{h} U\right|^{2}\right)^{(m-2) / 4}\left|\nabla_{h} U_{x_{1}}\right| \in l_{2}\left(\Omega_{h}\right)$ for $s=1,2$. Our constant $J_{3}$ is now estimated by the inequality

$$
\begin{aligned}
C_{2}^{2} J_{4} \leqq & 4 A\left\{2^{m^{\prime}}\left(C_{4}^{2}+\left(144 C_{3}^{2}+C_{6}^{2}+C_{5}^{\prime^{2}}\right)+C_{2}\left(C_{6}+C_{4}\right)\right\}\left(m_{h}\left(\Omega_{h}\right)+J_{1}^{m}\right)\right. \\
& +2 A C_{2} \max \left\{1,2^{\bar{m}}\right\} C_{5}^{\prime}\left(m_{h}\left(\Omega_{h}\right)+J_{1}^{m^{\prime}}\left(m_{h}\left(\Omega_{h}\right)\right)^{1 / m}\right),
\end{aligned}
$$

where $A=\left(\max \left\{\max |\eta|, \max \left|\nabla_{h} \eta\right|\right\}\right)^{2}$.

(b) If $m=2$, we have

$$
\begin{aligned}
C_{2}\left(|| \nabla_{h} U_{x_{0}} \mid \|_{2}^{0}\right)^{2} \leqq & 6 C_{3}\left\{h^{2} \sum \eta^{2} \epsilon_{1}\left|\nabla_{h} U_{x_{s}}\right|^{2}+h^{2} \sum\left|\nabla_{h} \eta\right|^{2}\left|\nabla_{h} U\right|^{2} / \epsilon_{1}\right\} \\
& +C_{5}^{\prime} h^{2} \sum \eta^{2}+4 h^{2} \sum\left(C_{6}\left|\nabla_{h} \eta\right| \eta\left|\nabla_{h} U\right|^{2}+C_{5}^{\prime} \eta\left|\nabla_{h} U\right|\right) \\
& +2 C_{4} h^{2} \sum \eta\left|\nabla_{h} \eta\right|\left(1+\left|\nabla_{h} U\right|^{2}\right) \\
& +C_{4} \epsilon_{2} h^{2} \sum \eta^{2}\left|\nabla_{h} U_{x_{s}}\right|^{2} / 2+C_{4} h^{2} \sum \eta^{2}\left(1+\left|\nabla_{h} U\right|^{2}\right) / 2 \epsilon_{2} .
\end{aligned}
$$

Now choose $\epsilon_{1}=C_{2} / 24 C_{3}$ and $\epsilon_{2}=C_{2} / 2 C_{4}$ to get the estimate

$$
\begin{aligned}
C_{2} J_{5} / 2 \leqq & 144\left(C_{3}^{2} / C_{2}\right) h^{2} \sum\left|\nabla_{h} \eta\right|^{2}\left|\nabla_{h} U\right|^{2} \\
& +C_{5}^{\prime} h^{2} \sum \eta^{2}+C_{4}^{2} h^{2} \sum \eta^{2}\left(1+\left|\nabla_{h} U\right|^{2}\right) / C_{2} \\
& +4 h^{2} \sum\left(C_{6}\left|\nabla_{h} \eta\right| \cdot\left|\nabla_{h} U\right|+C_{5}^{\prime}\right) \eta\left|\nabla_{h} U\right| \\
& +2 C_{4} h^{2} \sum \eta\left|\nabla_{h} \eta\right|\left(1+\left|\nabla_{h} U\right|^{2}\right) .
\end{aligned}
$$

(c) Now apply the Hölder Inequality to (10) to get

$$
\begin{aligned}
C_{2}\left(h^{2} \sum(1\right. & \left.\left.+\left|\nabla_{h} U\right|^{2}\right)^{m / 2}\right)^{\bar{m} / m}\left(h^{2} \sum \eta^{m}\left|\nabla_{h} U_{x_{s}}\right|^{m}\right)^{2 / m} \\
\leqq & \left(h^{2} \sum\left\{\left(12 C_{3}\left|\nabla_{h} \eta\right|+\eta C_{6}\right)\left(1+\left|\nabla_{h} U\right|^{2}\right)^{m^{\prime} / 2}\right\}^{m / m^{\prime}}\right)^{m^{\prime} / m}\left\|\eta\left(\nabla_{h} U_{x_{s}}\right)\right\|_{m}^{0} \\
& +\left(h^{2} \sum\left\{\eta\left(1+\left|\nabla_{h} U\right|^{2}\right)^{m^{\prime} / 2}\right\}^{m / m^{\prime}}\right)^{m^{\prime} / m}\left(h^{2} \sum\left(\eta\left|\nabla_{h} U_{x_{s}}\right|\right)^{m}\right)^{1 / m} \\
& +C_{5}^{\prime} h^{2} \sum \eta^{2}+4 C_{6} h^{2} \sum\left|\nabla_{h} \eta\right| \eta\left(1+\left|\nabla_{h} U\right|^{2}\right)^{m / 2} \\
& +4 C_{5}^{\prime} h^{2} \sum \eta\left|\nabla_{h} \eta\right|\left(1+\left|\nabla_{h} U\right|^{2}\right)^{m^{\prime} / 2} .
\end{aligned}
$$

Now apply the Schwartz Inequality to the first two terms on the right side of the above to get, taking $\epsilon_{1}=C_{2}\left(h^{2} \sum\left(1+\left|\nabla_{h} U\right|^{2}\right)^{m / 2}\right)^{\bar{m} / m} / 2$ and $\epsilon_{2}=\epsilon_{1}$,

$$
\begin{gathered}
\left(h^{2} \sum \eta^{m}\left|\nabla_{h} U_{x}\right|^{m}\right)^{2 / m} \leqq\left(2 / C_{2}\right)\left(h^{2} \sum\left(1+\left|\nabla_{h} U\right|^{2}\right)^{m / 2}\right)^{-\bar{m} / m} C_{5}^{\prime} h^{2} \sum \eta^{2} \\
+\left(h^{2} \sum\left(1+\left|\nabla_{h} U\right|^{2}\right)^{m / 2}\right)^{-\bar{m} / m} \\
\text { (12) } \quad \cdot\left\{\left(h^{2} \sum\left\{\left(12 C_{3}\left|\nabla_{h} \eta\right|+\eta C_{6}\right)\left(1+\left|\nabla_{h} U\right|^{2}\right)^{m^{\prime} / 2}\right\}^{m / m^{\prime}}\right)^{2 m^{\prime} / m}\right. \\
\left.+\left(h^{2} \sum\left\{\eta\left(1+\left|\nabla_{h} U\right|^{2}\right)^{m^{\prime} / 2}\right\}^{m / m^{\prime}}\right)^{2 m^{\prime} / m^{\prime}}\right\} / C_{2} \\
+4 C_{6} h^{2} \sum\left|\nabla_{h} \eta\right| \eta\left(1+\left|\nabla_{h} U\right|^{2}\right)^{m / 2}+4 C_{5}^{\prime} h^{2} \sum \eta\left|\nabla_{h} \eta\right|\left(1+\left|\nabla_{h} U\right|^{2}\right)^{m^{\prime} / 2} .
\end{gathered}
$$


(d) Now let $\xi(P) \in \delta_{3}$ and let $\phi(\xi(\cdot) ; P)$ be the solution to (5). We now derive an estimate on the norm of $\nabla_{h} \phi_{x_{0}}(\xi(\cdot) ; P)$ which is valid for all $\xi(P) \in S_{3}$.

In this case, we have

$$
\begin{aligned}
h^{2} \sum a_{i}\left(P, \xi, \nabla_{h} \phi\right) \zeta_{x_{i}} & =-h^{2} \sum\left(a_{i}\left(P, \xi, \nabla_{h} \phi\right)\right)_{x_{s}} \mu_{x_{i}} \\
& =-h^{2} \sum\left\{\tilde{a}_{i, p_{k}} \phi_{x_{k} x_{o}}+\tilde{a}_{i, \xi} \xi_{x_{s}}+\tilde{a}_{i, x_{s}}\right\} \mu_{x_{i}},
\end{aligned}
$$

where $\tilde{a}_{i, p k}, \tilde{a}_{i, \xi}$ and $\tilde{a}_{i, x}$ is defined as in (9) but now the arguments of the associated integrands are $\left(P, \xi, \nabla_{h} \phi\right)$ with $\Phi=(1-t) \phi\left(P-h_{s}\right)+t \phi(P),\left(P, \xi, \nabla_{h} \phi\left(P-h_{s}\right)\right)$ with $\xi=(1-t) \xi\left(P-h_{s}\right)+t \xi(P)$, and $\left(\tilde{P}, \xi(P-h), \nabla_{h} \phi\left(P-h_{s}\right)\right)$. Then, taking $\mu(P)=\eta^{2}(P) \phi_{x}$, assuming $m \geqq 2$ and $\kappa \geqq 0$ and setting $\tilde{m}=m-1-\kappa$,

$$
\begin{aligned}
C_{2} h^{2} \sum \eta^{2}(1 & \left.+\left|\nabla_{h} \phi\right|^{2}\right)^{(m-2) / 2}\left|\nabla_{h} \phi_{x}\right|^{2} \\
\leqq & h^{2} \sum\left(6 C_{3} \eta^{2} \epsilon_{1}+\eta^{2} C_{6} \epsilon_{2} / 2+\eta^{2} C_{5}^{\prime} \epsilon_{3} / 2\right)\left(1+\left|\nabla_{h} \phi\right|^{2}\right)^{\bar{m} / 2}\left|\nabla_{h} \phi\right|^{2} \\
& +C_{4}\left(h^{2} \sum\left(1+\left|\nabla_{h} \xi\right|^{2}\right)^{\tilde{m}}\right)^{1 / 2}\left(h^{2} \sum \eta^{4}\left|\nabla_{h} \phi_{x}\right|^{2}\right)^{1 / 2} \\
& +h^{2} \sum 6 C_{3}\left|\nabla_{h} \eta\right|^{2}\left(1+\left|\nabla_{h} \phi\right|^{2}\right)^{m / 2} \epsilon_{1} \\
& +C_{6}\left(h^{2} \sum\left|\nabla_{h} \xi\right|^{m}\right)^{2 / m}\left(h^{2} \sum \eta^{2 m / m}\left(1+\left|\nabla_{h} \phi\right|^{2}\right)^{m / 2}\right)^{\bar{m} / m} / 2 \epsilon_{2} \\
& +C_{5}^{\prime} h^{2} \sum \eta^{2}\left(1+\left|\nabla_{h} \phi\right|^{2}\right)^{\bar{m} / 2} / 2 \epsilon_{3}+4 h^{2} \sum C_{5}^{\prime} \eta\left(1+\left|\nabla_{h} \phi\right|^{2}\right)^{m^{\prime} / 2} \\
& +4 C_{6}\left(h^{2} \sum \eta^{m / m}\left(1+\left|\nabla_{h} \phi\right|^{2}\right)^{m / 2}\right)^{m^{\prime} / m}\left(h^{2} \sum\left|\nabla_{h} \eta\right|^{m}\left|\nabla_{h} \xi\right|^{m}\right)^{1 / m} \\
& +2 C_{4}\left(h^{2} \sum \eta^{m}\left|\nabla_{h} \phi\right|^{m}\right)^{1 / m}\left(h^{2} \sum\left|\nabla_{h} \eta\right|^{m / m^{\prime}}\left(1+\left|\nabla_{h} \xi\right|^{2}\right)^{\tilde{m} / m^{\prime}}\right)^{m^{\prime} / m} .
\end{aligned}
$$

Now choose $\epsilon_{1}=C_{2} / 48 C_{3}, \epsilon_{2}=C_{2} / 16 C_{6}, \epsilon_{3}=C_{2} / 16 C_{5}^{\prime}, \epsilon_{4}=C_{2} / 16 C_{4}$ and observe that for $p>2$ we have $\eta^{p} \leqq \eta^{2}$ whenever $\eta \leqq 1$ to get an estimate on $J_{8}$ from (13).

(e) If $4 / 3 \leqq m<2$, then we use (13) and the Schwartz Inequality to obtain an estimate.

Remark 3. Parts (d) and (e) of the last theorem are stated in their present generality so that we may show the exact dependence of the norms of $\left|\nabla_{h} \phi_{x}\right|$ on the properties of the coefficients.

In our next result we prove that if $\Omega$ is a rectangular region, then our "interior estimates" may hold for all of $\Omega_{h}$.

COROLlARY 2. If $\Omega$ is a rectangular domain with vertices $(0,0),(a, 0),(a, b),(0, b)$, if $a / b$ is rational and $h$ divides $a$ and $b$, if $a_{1}\left(P, U, \nabla_{h} U\right)=0$ for $P \in s_{2}+s_{4}$ and $a_{2}\left(P, U, \nabla_{h} U\right)=0$ for $P \in s_{1}+s_{3}$, if conditions (B), (C) and (D) are satisfied, and if $U(P)$ is a solution to (3) in $H_{m, 0}^{1}\left(\Omega_{h}\right)$ with $m \geqq 2$, then there exists a positive constant $J_{11}$ such that, for $s=1$ and $s=2$,

$$
h^{2} \sum\left(1+\left|\nabla_{h} U\right|^{2}\right)^{\bar{m} / 2}\left|\nabla_{h} U_{\dot{x}_{1}}\right|^{2} \leqq J_{11}
$$

where $J_{11}$ depends on $\left\|\nabla_{h} U\right\|_{m}$ and the material constants of our conditions.

Proof. Let $\zeta=U_{x_{1 \neq} x_{1}}$ where we have reflected $U$ as an odd function. Then, integrating by parts in the $x_{1}$-direction, we get the identity

$$
h^{2} \sum_{\Omega_{h}}\left\{a_{i, \dot{x}_{1}} U_{\tilde{x}_{1} x_{i}}+f U_{x_{1} \dot{x}_{1}}\right\}=0 .
$$

Proceeding as in the development of (9), we get

$$
h^{2} \sum_{\mathbb{R}_{h^{\prime}}}\left\{\tilde{a}_{i, p_{j}} U_{\tilde{x}_{1} x_{i}} U_{\tilde{x}_{1} x_{i}}+\tilde{a}_{i, u} U_{\hat{x}_{1}} U_{\tilde{x}_{1} x_{i}}+\tilde{a}_{i, x_{1}} U_{\tilde{x}_{1} x_{1}}+f U_{x_{1} x_{1}}\right\}=0
$$


and hence, for $\epsilon_{i}>0$ with $i=1,2$, we have

$$
\begin{aligned}
& C_{2} h^{2} \sum\left(1+\left|\nabla_{h} U\right|^{2}\right)^{\bar{m} / 2}\left|\nabla_{h} U_{\dot{x}_{1}}\right|^{2} \\
& \leqq h^{2} \sum\left(1+\left|\nabla_{h} U\right|^{2}\right)^{\bar{m} / 2}\left\{C_{6}\left|\nabla_{h} U\right|+C_{5}\right\}\left|\nabla_{h} U_{\dot{x}_{1}}\right| \\
&+h^{2} \sum C_{4}\left(1+\left|\nabla_{h} U\right|^{2}\right)^{m^{\prime} / 2}\left|\nabla_{h} U_{x_{1}}\right| \\
& \leqq\left(\left(\epsilon_{1}+\epsilon_{2}\right) / 2\right) h^{2} \sum\left(1+\left|\nabla_{h} U\right|^{2}\right)^{\bar{m} / 2}\left|\nabla_{h} U_{\tilde{x}_{1}}\right|^{2} \\
&+\left(C_{4}^{2} / 2 \epsilon_{2}+C_{5}^{2} / \epsilon_{1}+C_{6}^{2} / \epsilon_{1}\right) h^{2} \sum\left(1+\left|\nabla_{h} U\right|^{2}\right)^{m / 2}
\end{aligned}
$$

Now we shall prove the convergence of solutions of the difference equations to weak solutions of the differential equation. Our proof will make essential use of our interior estimates and the fact that our equation has two independent variables. A different convergence proof, with less stringent hypotheses, is to be found in Frehse [3, p. 331].

Let $\Omega$ be a domain with the $\partial \Omega$ in $C^{1}$. Let $D, D^{\prime}$ be domains with $\partial D, \partial D^{\prime}$ in $C^{1}$ and such that $\bar{D}^{\prime} \subset D$ and $\bar{D} \subset \Omega$. Let $h_{n}$ be a sequence of positive numbers tending monotonically to zero such that $\Omega_{h_{n+1}} \supset \bar{\Omega}_{h_{n}}$. Let $h^{\prime}>0$ be such that for $n \geqq N\left(h^{\prime}\right)$ we have that $D_{h_{n}} \supset D^{\prime}$ and $\Omega \supset \bar{D}_{h_{n} \text {. }}$.

Assume the appropriate-we have not yet made an assumption on $m$-hypotheses of Theorem 1 are satisfied so that a solution, $U_{n}(P) \in a_{0}\left(\Omega_{h}\right)$, exists to the difference equation

$$
h_{n}^{2} \sum_{P \in \Omega_{h_{n}}}\left\{a_{i}\left(P, U_{n}(P), \nabla_{h} U_{n}(P)\right) \zeta_{x_{i}}(P)+f\left(P, U_{n}(P), \nabla_{h} U_{n}(P)\right) \zeta(P)\right\}=0
$$

for all $\zeta(P) \in Q_{0}\left(\Omega_{h}\right)$. Let $\mathcal{u}_{n}(P)$ be the "filling-in" function given in Stummel [15, p. 180]; i.e.

$$
\mathfrak{u}_{n}(P)=h_{n}^{2} \sum_{Q} S_{h}(P-Q) U_{n}(Q),
$$

where $Q$ runs over all mesh points of the plane and

$$
S_{h}(P-Q)=\left[\sin \left(\pi\left(x_{1}-\xi_{1}\right)\right) / \pi\left(x_{1}-\xi_{1}\right)\right]\left[\sin \left(\pi\left(x_{2}-\xi_{2}\right)\right) / \pi\left(x_{2}-\xi_{2}\right)\right]
$$

with $P=\left(x_{1}, x_{2}\right)$ and $Q=\left(\xi_{1}, \xi_{2}\right)$.

By Theorem 3, we have that $U_{n}(P) \in H_{2}^{2}\left(D_{h_{n}}\right)$ or we have $U_{n}(P) \in H_{m}^{2}\left(D_{h}\right)$ depending on the size of $m$. Let us assume $m \geqq 2$. Then there exists a constant independent of $h_{n}$ such that $\left\|U_{n}(P)\right\|_{2}^{2} \leqq J_{4}^{\prime}$ for each $n \geqq N\left(h^{\prime}\right)$ and this norm is taken over $D_{h_{n}}$. Hence, there exists a constant $J_{4}^{\prime \prime}$, independent of $h_{n}$, such that $\left\|\mathcal{u}_{n}(P)\right\|_{2}^{2} \leqq J_{4}^{\prime \prime}$ where this norm is over $D^{\prime}$; see Stummel [15, p. 181]. Applying the Variant of the Calderon Extension Theorem [13, p. 74], we have new functions $\mathcal{V}_{n}(P) \in \mathcal{F C}_{2,0}^{2}(\Omega)$ such that $\vartheta_{n}(P)=\mathcal{U}_{n}(P)$ in $D^{\prime}$; these functions are also uniformly bounded over $\Omega$ in the $\mathcal{F}_{2,0}^{2}(\Omega)$ norm. Hence, a subsequence of $V_{n}$, which we still call $V_{n}$, converges weakly to some $\mathcal{V}_{0}(P) \in \mathcal{F C}_{2,0}^{2}(\Omega)$. Using Theorem 3.2.3 in [12, p. 70] and Theorem 10.2 in $\left[4\right.$, p. 28], we conclude that a subsequence of $V_{n}$ converges strongly to $V_{0}$ in $\mathcal{F}_{m, 0}^{1}(\Omega)$. Since $\mathcal{F}_{m, 0}^{1}(\Omega)$ and $H_{m, 0}^{1}\left(\Omega_{h}\right)$ are conditionally compact with respect to weak convergence, the above analysis shows that if a subsequence of $\mathcal{U}_{n}$, which we still call $\mathfrak{u}_{n}$, converges weakly to an element $\mathcal{u}_{0} \in \mathcal{F C}_{m, 0}^{1}(\Omega)$, then for any set $D^{\prime} \subset \Omega$ satisfying the conditions above, we have that $\mathcal{U}_{n}$ converges strongly to $\mathcal{U}_{0}$ in $\mathcal{F}_{m}^{1}\left(D^{\prime}\right)$.

We now claim that, for all $\zeta \in C_{c}^{1}\left(D^{\prime}\right)$, 


$$
\iint_{\Omega}\left\{a_{i}\left(P, \mathcal{V}_{0}(P), \nabla \mathcal{V}_{0}(P)\right) \zeta_{x_{i}}(P)+f\left(P, \mathcal{V}_{0}(P), \nabla_{h} \mathcal{V}_{0}(P)\right) \zeta(P)\right\} d x_{1} d x_{2}=0 .
$$

To see this we first observe that $\mathcal{u}_{n}(P)=U_{n}(P)$ for $P \in \Omega_{h_{n}}$ and that, using the methods of proof in [15, pp. 186-187], derivatives and difference quotients of $\mathfrak{u}_{n}(P)$ converge strongly to $\mathcal{U}_{0}(P)$ in $\mathcal{H}_{m}^{1}\left(D^{\prime}\right)$. Now, to each $P \in \Omega_{h_{n}}$ with $n>N\left(h^{\prime}\right)$ let us associate the rectangular region $\Delta_{n}(P)$ determined by the vertices $\left(x_{1}, x_{2}\right),\left(x_{1}+h, x_{2}\right)$, $\left(x_{1}+h, x_{2}+h\right),\left(x_{1}, x_{2}+h\right)$. Over $\Delta_{h}(P)$ let us define $\bar{O}_{n}(Q)=U_{n}(P)$ and $\nabla_{h} D_{n}(Q)=\nabla_{h} U_{n}(P)$ for all $Q \in \Delta_{n}(P)$. Now we observe that there exists $\epsilon_{i}(n)$ for $i=1,2$ such that $\epsilon_{i}(n) \rightarrow 0$ as $n \rightarrow \infty$ and

$$
\begin{aligned}
\iint_{\Delta_{n}(P)} a_{i}(Q, & \left.\bar{U}_{n}, \nabla_{h} U_{n}\right) \zeta_{x_{i}}(Q) d Q \\
& =\left\{\zeta_{x_{i}}(P)+\epsilon_{i}(n)\right\} h_{n}^{2} a_{i}\left(P, U_{n}, \nabla_{h} U_{n}\right)+O\left(h_{n}^{3}\right)\left(1+\epsilon_{i}(n)\right) .
\end{aligned}
$$

Also, from the strong convergence derived above and the fact that $\mathcal{U}_{n}(P)=U_{n}(P)$ for $P \in \Omega_{h}$ and the appropriate conditions in (A) to (E), we deduce the result that

$$
\iint_{\Omega}\left\{a_{i}\left(Q, U_{n}, \nabla_{h} U_{n}\right)-a_{i}\left(Q, \mathfrak{u}_{n}(Q), \nabla \mathfrak{u}_{n}(Q)\right)\right\} \zeta_{x_{i}}(Q) d Q \rightarrow 0 \quad \text { as } n \rightarrow \infty .
$$

In a similar manner, we have that

$$
\iint_{\Delta_{n}} f\left(Q, U_{n}, \nabla_{h} U_{n}\right) \zeta(Q) d Q=h_{n}^{2} f\left(P, U_{n}, \nabla_{h} U_{n}\right) \zeta(P)+\epsilon(n)
$$

and

$$
\iint_{\Omega}\left\{f\left(Q, U_{n}, \nabla_{h} U_{n}\right)-f\left(Q, \mathfrak{u}_{n}(Q), \nabla \mathfrak{u}_{n}(Q)\right)\right\} \zeta(Q) d Q \rightarrow 0 \quad \text { as } n \rightarrow \infty ;
$$

here $\epsilon(n) \rightarrow 0$ as $n \rightarrow \infty$ and comes from Condition (E) and $\zeta \in C_{c}^{1}\left(D^{\prime}\right)$. Using the fact that $U_{n}(P)$ solves the difference equation, the additivity of the integral, and the linearity of $\zeta$ and $\nabla \zeta$ in the integral, we conclude that $\mathcal{u}_{0}(P)$ is a weak solution of (1). The case $m \in[4 / 3,2]$ proceeds along similar, but simpler, lines. Therefore, we have proved the next result.

THEOREM 5. Let $h_{n}$ be a monotonically decreasing sequence which converges to zero and such that $\Omega_{h_{n+1}} \supset \bar{\Omega}_{h_{n}}$. Let the $\partial \Omega$ be in $C^{1}$. Let the appropriate hypotheses of Theorem 1 and Theorem 3 be satisfied. Let $U_{n}(P)$ be the discrete solutions to (3) with $h=h_{n}$ and let $\mathcal{u}_{n}(P)$ be as in (14). Then, there exists a subsequence of $\mathcal{u}_{n}(P)$ and an element $\mathfrak{U}_{0}(P) \in \mathfrak{H}_{m, 0}^{1}(\Omega)$ such that $\mathcal{U}_{n}(P)$ converges weakly to $\mathcal{U}_{0}(P)$ in $\mathcal{F C}_{m, 0}^{1}(\Omega), \mathcal{U}_{n}(P)$ converges strongly to $\mathfrak{U}_{0}(P)$ in $\mathcal{H}_{m}^{1}(D)$ with $\partial D$ in $C^{1}$ and $\bar{D} \subset \Omega$ and the function $\mathcal{U}_{0}(P)$ is a weak solution to the differential equation (1); i.e. (15) holds for every $\zeta(P) \in \mathcal{H}_{m, 0}^{1}(\Omega)$.

As an immediate consequence of the last result and that in Corollary 2 we have the following.

COROLlARY 3. Let $\Omega$ be a rectangular region and let $h_{n}$ tend monotonically to zero with $h_{n}>0, \Omega_{h_{n+1}} \supset \Omega_{h_{n}}$ and $\partial \Omega_{h_{n+1}} \supset \partial \Omega_{h_{n}}$. Let $U_{n}(P)$ be a solution to (3) and let $\mathcal{U}_{n}(P)$ be as given in (14). Let the hypotheses of Corollary 2 be satisfied. Then there exists an element $\mathfrak{u}_{0}(P) \in \mathcal{F}_{2,0}^{2}(\Omega)$ such that some subsequence of $\mathfrak{u}_{n}(P)$ converges 
weakly in $\mathcal{C}_{2,0}^{2}(\Omega)$ to $\mathfrak{U}_{0}(P)$ and a further subsequence of $\mathfrak{U}_{n}(P)$ converges strongly to $\mathcal{U}_{0}(P)$ in $\mathfrak{H}_{m, 0}^{1}(\Omega)$. This function $\mathcal{U}_{0}(P)$ satisfies $(15)$.

Remark 4. From results in [12, pp. 78-81], [1] and [13], we may use estimates on norms of second-order difference quotients over all of $\Omega_{h}$ to prove the pointwise convergence of solutions of $(* * *)$ to weak solutions of the differential equation. These same techniques also work using estimates of the $H_{m, 0}^{1}\left(\Omega_{h}\right)$ norm of solutions to (3) provided $m$ is sufficiently large relative to the number of independent variables; see $[12$, p. 83].

(II). The Case that $Q(P) \not \equiv 0$ for $P \in \Omega_{h}$. We shall assume that there exists a function $\tilde{q}(P) \in C^{2}(\bar{\Omega})$ such that $Q(P)=\tilde{q}(P)$ for $P \in \bar{\Omega}_{h}$ and $\tilde{q}(P)=q(P)$ for $P \in \partial \Omega$.

Now we reformulate (14) slightly. We seek a mesh function $U(P) \in Q_{0}\left(\Omega_{h}\right)$ such that for all $\zeta(P) \in Q_{0}\left(\Omega_{h}\right)$ we have

$$
h^{2} \sum_{\Omega_{h}}\left\{a_{i}\left(P, U+Q, \nabla_{h}(U+Q)\right) \zeta_{x_{i}}+f\left(P, U+Q, \nabla_{h}(U+Q)\right) \zeta\right\}=0 .
$$

It is clear that all of the estimates we have obtained in (1) go through for (16) with slight modification. These new bounds will clearly depend on discrete $l_{m}$-norms of $Q(P)$ and its difference quotients.

We may extend our data to the case that $q(P)$ has a continuation $\tilde{q}(P)$ into $\Omega$ such that $\tilde{q}(P) \in \mathcal{F}_{2}^{2}(\Omega)$. Our analysis in this case would follow that of our proof of convergence.

Center for the Application of Mathematics

Lehigh University

Bethlehem, Pennsylvania 18015

1. R. Courant, K. O. Friedrichs \& H. Lewy, "Über die partiellen Differenzengleichungen der mathematischen Physik," Math. Ann., v. 100, 1928, pp. 32-74; English transl., IBM J., v. 11, 1967, pp. 215-234.

2. C. W. CRYER, "The difference analogue of Gauss' theorem," SIAM J. Numer. Anal. v. 4, 1967, pp. 155-162. MR 35 \#4620.

3. J. FreHSE, "Existenz und Konvergenz von Lösungen nichtlinearer elliptischer Differenzengleichungen unter Dirichlet-Randbedingungen," Math. Z., v. 109, 1969, pp. 311-343. MR 40 \#1037.

4. Avner Friedman, Partial Differential Equations, Holt, Rinehart and Winston, New York, 1969.

5. N. N. Gudovič, "The application of difference methods to the solution of nonlinear elliptic equations," Dokl. Akad. Nauk SSSR, v. 179, 1968, pp. 1257-1260 = Soviet Math Dokl., v. 9, 1968, pp. 525-528. MR 37 \#389.

6. W. VON KOPPENFELS, Über die Existenz der Lösungen linearer partieller Differentialgleichungen vom elliptischen Typus, Dissertation, Göttingen, 1929.

7. O. A. LADYŽENSKAYA, "The method of finite differences in the theory of partial differential equations," Uspehi Mat. Nauk, v. 12, 1957, pp. 123-148; English transl., Amer. Math. Soc. Transl., (2), v. 20, 1962, pp. 77-104, MR 25 \#342.

8. O. A. LADYŽENSKAYA \& N. N. URAL'CEVA, Linear and Quasilinear Differential Equations of Elliptic Type, "Nauka," Moscow, 1964; English transl., Academic Press, New York, 1968. MR 35 \#1955; MR 39 \#5941.

9. G. T. MCALLISTER, "An application of a priori bounds on difference quotients to a constructive solution of mildly quasilinear Dirichlet problems," J. Math. Anal. Appl., v. 24, 1968, pp. 582-607. MR 38 \#2963.

10. G. T. MCALlister, "Dirichlet problems for mildly nonlinear elliptic difference equations," J. Math. Anal. Appl., v. 27, 1969, pp. 338-366. MR 40 \#7659.

11. C. B. MoRrey, JR., "Multiple integral problems in the calculus of variations and related topics," Univ. California Publ. Math., v. 1, 1943, pp. 1-130. MR 6, 180.

12. C. B. MoRREY, JR., Multiple Integrals in the Calculus of Variations, Die Grundlehren der math. Wissenschaften, Band 130, Springer-Verlag, New York, 1966. MR 34 \#2380. 
13. I. G. Petrowsky \& K. N. Smirnoff, "Sur une condition suffisante pour qu'une famille de fonctions soit également continue," Bull. Univ. Moscow Sect. A Math. Mech., v. 10, 1938, pp. 1-15.

14. S. L. Sobolev, "Sur l'évaluation de quelques sommes pour une fonction définie sur un réseau," Izv. Akad. Nauk SSSR, v. 4, 1940, pp. 5-16. MR 1, 298.

15. FRIEDRICH STUMMEL, "Elliptische Differenzenoperatoren unter Dirichlet-Randbedingungen," Math. Z., v. 97, 1967, pp. 169-211. MR 36 \#7346.

16. B. A. VERTGEIM, "On the approximate determination of the fixed points of continuous mappings," Dokl. Akad. Nauk SSSR, v. 191, 1970, pp. 9-11 = Soviet Math. Dokl., v. 11, 1970, pp. 295-298. 